\title{
A novel method for evaluating intake unsteady flow distortion
}

\author{
Matteo Migliorini' ${ }^{1}$, Pavlos K. Zachos ${ }^{2}$, and David G. MacManus ${ }^{3}$ \\ Propulsion Engineering Centre, School of Aerospace Transport and Manufacturing, \\ Cranfield University, MK43 OAL, Cranfield, UK
}

Closer integration between the airframe and the propulsion system is expected for future aircraft to reduce fuel consumption, emissions, weight and drag. The use of embedded or partially embedded propulsion systems will require the use of complex intakes. However, this can result in unsteady flow distortion which can adversely affect the propulsion system efficiency and stability. Relative to conventional measurement systems, time-resolved Particle Image Velocimetry provides sufficient spatial and temporal resolution to enable the development of new methods to assess unsteady flow distortion. This work proposes a novel analysis approach to assess the unsteady flow distortion. For an S-duct configuration, the method was successfully used to evaluate the unsteady flow distortion in terms of idealized incidence angle perturbations. This example showed peaks up to $\pm 30^{\circ}$ incidence and a duration equivalent to the passing time of 3 blades. The introduction of a non-uniform total pressure profile at the S-duct inlet increased the probability of peak distortion events with higher magnitude. The method provides an estimate of the likelihood, magnitude and duration of distortion events and is a new way to evaluate flow distortion that could induce instabilities for the propulsion system.

${ }^{1}$ PhD Student, Propulsion Engineering Centre, Member AIAA.

${ }^{2}$ Senior Lecturer, Propulsion Engineering Centre, Member AIAA.

${ }^{3}$ Professor, Propulsion Engineering Centre, Member AIAA.

Partially presented as Paper 2019-4201 at the AIAA Propulsion and Energy Forum and Exposition, Indianapolis, IN, August 19-22, 2019. 


\section{Nomenclature}

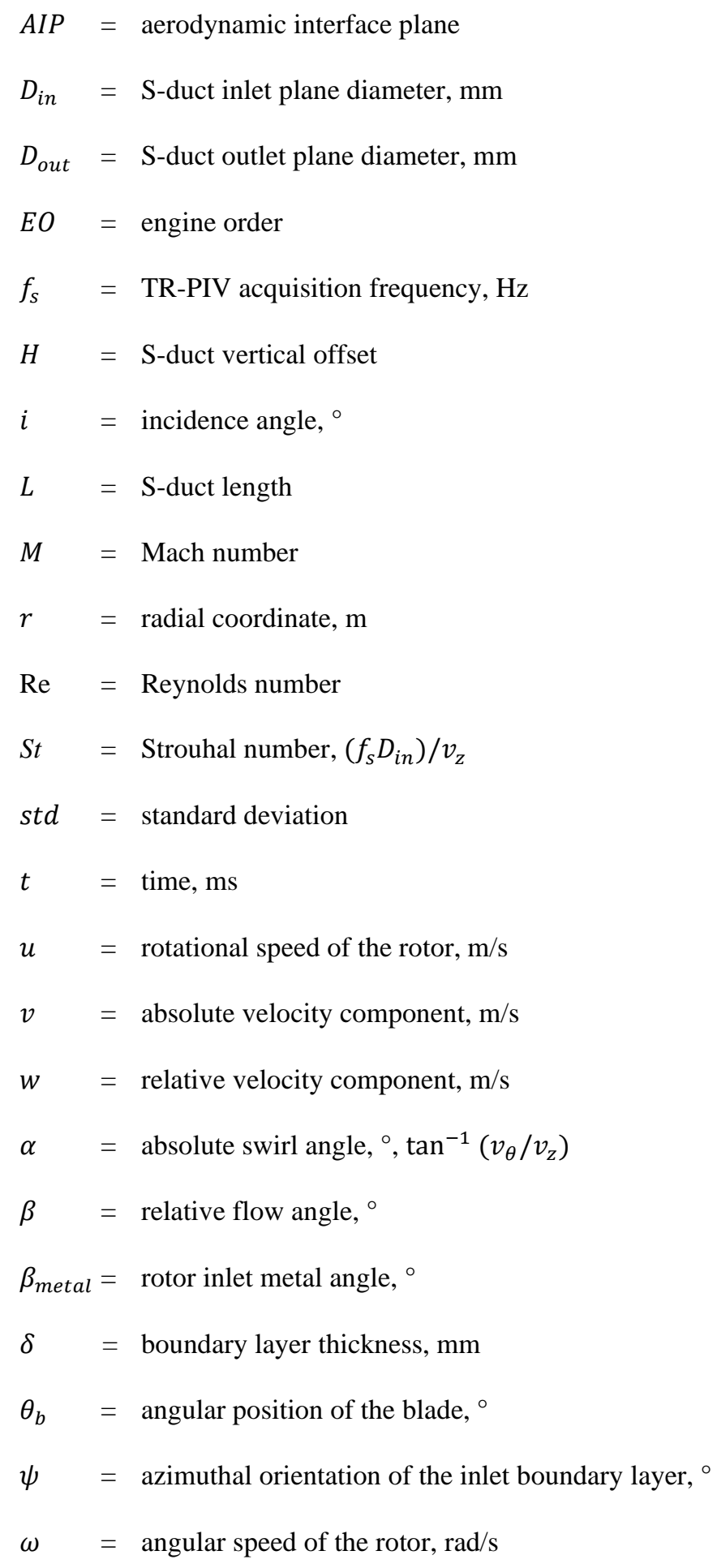




\section{Operators}

$$
\begin{aligned}
\langle\rangle & =\text { time average } \\
- & =\text { area average }
\end{aligned}
$$

\section{Introduction}

$\mathrm{U}$ NDERSTANDING the response of an aero-engine compressor system to inlet flow distortion can be a crucial requirement for the integration of the propulsion system with the airframe. Since the 1960s, many researchers have simulated the inlet distortion numerically and experimentally and observed a reduction of the engine performance [1]. Experiments on engine stability monitored the compressor pressure ratio when the inlet was exposed to different sectors of pressure distortion, and it was found that sectors of circumferential total pressure loss of $60^{\circ}$ to $90^{\circ}$ can reduce the engine surge margin. Within this context, industry guidelines [2] suggest that a $25^{\circ}$ sector is a minimum critical angle for the extent of a total pressure distortion. Further research has established the critical disturbances for the engine stability and to identify the key frequencies and extent signatures [3]. Rather than a single critical frequency, a range of disturbance frequencies was identified to which the engine may respond. These studies showed that disturbances with a signature frequency of about 1 Engine Order (EO) could reduce the compressor surge margin [4] whereas the engine may respond in a quasi-steady manner to unsteady disturbances with a frequency substantially lower than 1EO. More recently, the response time for a disturbance to convect from the blade leading edge to the throat of the blade passage was evaluated by Cousins [3]. As shown by Cousins, for axial compressors, a disturbance extending across 4 to 5 blades may adversely affect the stability limit of the compression system [3]. Frequencies of the order of $1 \mathrm{EO}$ are also of interest as they could excite the first resonant frequency of the compressor rotor blades, which is typically $\mathrm{St}=0.7$ for a model scale compressor [5].

Cousins [3] considered the reduced frequency and concluded that intake and fan are coupled systems and that the impact of the inlet distortion on the compressor fan cannot be addressed by considering the two elements separately. Moreover, the studies by Hodder [6], and Larkin and Schweiger [7] indicated that the fan has an influence on the upstream flow distortion pattern. In these studies on large turbofan engines, the presence of the fan attenuated the instabilities arising from the intake lip separations and increased the operability range of the inlet beyond the separation angle-of-attack which was found when the fan was not included in the experiment. Similarly, also more recent studies 
by Carnevale et. al [8] confirmed that a fan acts as a flow distortion suppressor and thus can increase the tolerance to the incoming flow incidence.

Latest research revealed that also the unsteadiness of the flow can play a notable role on the engine stability. Shaw [9] demonstrated that for frequencies lower than 1 engine order, the fan is significantly less tolerant to a continuous stream of pulses rather than a single one. Similarly, a single reduction in total pressure may not be sufficient to trigger stall inception in transonic rotors and up to 2 full revolutions may be needed to fully develop a rotating stall [10]. There is also evidence of unsteady conditions generated by stators, whose wake passing changes periodically the incidence angle and the vorticity content. These periodic unsteady conditions can influence the spill-over of the tip clearance flow and for operating conditions near the stability limit these could trigger the rotating stall inception process [11]. Moreover, experiments with large turbofan engine revealed that rotating stall can be triggered by unstable oscillations that are caused by the delay between the response of the inlet and of the fan to incoming fluctuations of low frequency [12]. The spectral gap between the unsteadiness of the separation at the intake lip and the blade passing frequency could ultimately provoke resonance and a substantial drop in pressure ratio [13].

These long-length-scale disturbances are typical of one of the stall inception mechanisms, known as modal stall inception [14]. On the other hand, short-length-scale disturbances can also be a source of instability, since they could trigger the spike-type inception [15]. Wenzel and Blaba [16] showed that compressor stability was sensitive to disturbance duration rather than shape and that the greater impact on surge margin reduction was caused by shorter pulses. Silkowski's experiment [17] showed that a velocity disturbance of a length scale of 3 blade pitches and an amplitude deficit of $30 \%$ of the rotor tip speed triggered the stall inception. However, there is no general rule to predict the engine response for different inlet distortion. Published research indicates a typical range between 1-per-rev and blade passing frequency for disturbances that could contribute to the instability of a compression system.

Recently, there has been an increasing interest in engine stability assessments for closely-coupled propulsion systems for novel aircraft configurations. The integration of the propulsion system with convoluted intakes results in total pressure and swirl distortion, which can be critical for the engine operation and can cause stall inception and degradation of the propulsion system performance. Stereo Particle Image Velocimetry (SPIV) has been used to characterize the flow distortion of convoluted intakes in the work of Zachos et al. [18]. SPIV provided synchronous, non-intrusive measurements to characterize the swirl distortion and the flow unsteadiness with a spatial resolution significantly greater than that from conventional pressure transducer arrangements [19]. The dynamic and steady swirl 
distortion was found to be more pronounced in S-ducts with an high centerline offset [20]. The most energetic coherent structures, determined using proper orthogonal decomposition [21], showed that the flow is governed by a lateral swirl switching mode and a vertical mode. These flow modes result in the alternation of bulk swirl events and twin swirl events at the aerodynamic interface plane [20]. Investigations on how to reduce the peak swirl unsteadiness with vortex generators were reported by Tanguy et al. [22]. These devices suppressed the primary unsteady flow switching mechanism and the associated extreme events that lead to high levels of swirl intensity. More recently, time-resolved particle image velocimetry (TR-PIV) was used to assess the frequencies associated with the flow distortion modes [23]. The frequencies of these coherent structures are contained within $\mathrm{St}<1.2$; and therefore, they are within the range of the critical frequencies for a typical propulsion system. The S-duct aerodynamics were also investigated for non-uniform inlet conditions representative of possible approaching conditions of boundary layer ingestion airframe or intake operation under angles of yaw and pitch [24]. An intensification of the vortical structures and an increase of the swirl angle unsteadiness were observed with thicker inlet total pressure profiles.

Previous investigations have focused on two main areas of research: flow distortion measurements at the exit of an intake [25] and compressor system response for particular distortion configurations [26,27]. Out of necessity, the conventional metrics, which are used to characterize the flow distortion, have been constructed on the basis of the available measurement techniques. This is usually based on total pressure measurements taken with a fixed rake in the absence of the fan. These data were used to develop assessments of fan responses to different levels of total pressure and swirl distortion $[2,28]$. However, there is a notable gap between knowledge of total pressure distortion in the absolute frame of reference and the impact of unsteady flow distortion from the perspective of the rotating compressor system. It is arguable that without characterizing the approaching unsteady flow distortion in the compressor relative frame of reference, the pertinent underpinning distortion characteristics, which influence the stability of the compression system, are unlikely to be understood. Consequently, the state of the art is still highly dependent on bespoke correlations for a particular compressor system based on distortion data in the absolute frame of reference.

Within this context, the aim of the current work is to introduce a novel method for evaluating the unsteady flow distortion of aero-engine intakes. The objective is to propose a new technique for evaluating unsteady flow distortion which exploits the high spatial and temporal resolution of the unsteady three-component velocity distortion measurements obtained with TR-PIV $[18,22,23,29]$. The method considers the approaching unsteady flow distortion 
in the relative frame of reference of a notional compressor system. This synchronously assesses the spatial and temporal non-uniformities due to the unsteady flow field as well as the relative position and exposure of a set of envisaged rotor blades. The method is applied to a relatively simple S-duct configuration in which the effect of the hub and fan are not included. Thus, it is acknowledged that the reported levels of swirl distortion reflect the simplified experimental arrangement, and that the interactions between fan and the inlet distortion is not considered. However, the new approach and analysis methods presented in the paper would be equally applicable on experiments which include a fan stage or engine. The characterization and probabilistic analysis of the unsteady signatures of approaching distortion onto the compressor fan would be similarly applicable. It is envisaged that this method will open the route to the evaluation of the dynamic distortions through the circumferential modal analysis and the evaluation of radial extent and synchronicity of the distortion events.

\section{II.Experimental setup and methods}

\section{A. Experimental facility and S-duct configuration}

Although several boundary layer ingestion (BLI) configurations with flush mounted intakes with minimum or no offset have been proposed in the literature, some new civil and non-civil aircraft configurations are expected to employ more aggressive offset intakes. These include BLI configurations with relatively high S-duct offsets [26], aircrafts with dorsal mounted intakes [30] and embedded gas turbine intakes for the core engine in propulsive fuselage configurations. The S-duct configuration used in this study was previously investigated by Zachos et al. [18]. The test rig layout is shown in Fig. 1. Upstream of the S-duct intake, the facility comprises a seeding chamber, a flow conditioning section, a honeycomb screen station and a tubular duct of constant inlet diameter. The geometrical characteristics of the S-duct are shown in Table 1. This S-duct geometry was chosen as there is a substantial amount of previous work on this specific baseline configuration within the peer-reviewed journal literature. This encompasses experimental and computational studies $[29,31]$ as well as evaluations of steady and unsteady total pressure distortion [19], swirl distortion [23], sensitivity to inlet Mach number [18], as well as the effectiveness of passive and active flow control methods [22]. Downstream of the optical section, a diffuser duct drives the flow to the suction fan (Fig. 1). The operating point of the rig is set by the velocity of the flow measured in the section upstream the S-duct, at $1.45 \mathrm{D}_{\text {in }}$ upstream of the S-duct entry plane. For the current work, the reference Mach number was $\mathrm{M}_{\text {ref }}=0.27$ and the reference Reynolds number based on the inlet diameter was $\operatorname{Re}_{\mathrm{D}}=7.38 \times 10^{5}$. 


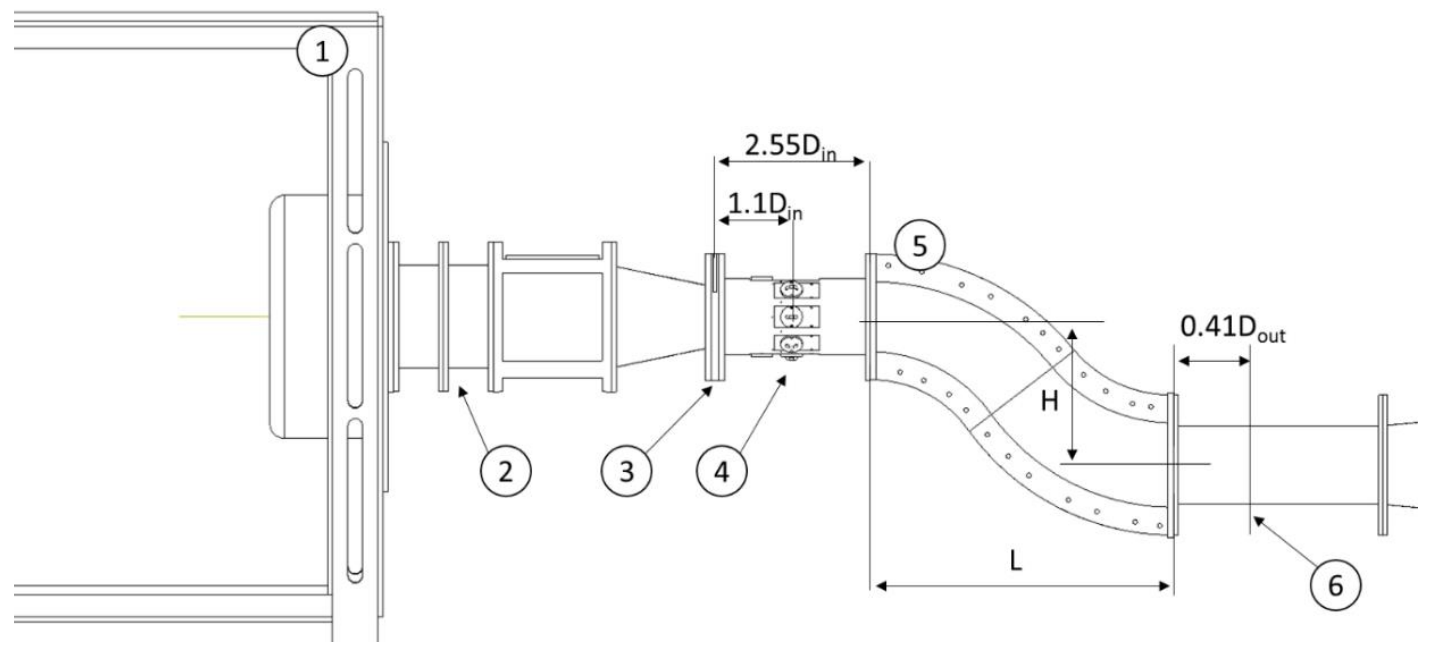

Fig. 1: Test facility layout: 1- seeding chamber, 2- flow conditioning section, 3- honeycomb screen section, 4- pressure measurements ports, 5- S-duct, and 6- PIV measurement plane.

Table 1: S-duct geometrical characteristics

\begin{tabular}{cc}
\hline \hline Parameter & High Offset S-duct \\
\hline $\mathrm{A}_{\text {out }} / \mathrm{A}_{\text {in }}$ & 1.52 \\
$\mathrm{~L} / \mathrm{D}_{\text {in }}$ & 4.95 \\
$\mathrm{H} / \mathrm{L}$ & 0.49 \\
$\mathrm{D}_{\text {out }}[\mathrm{mm}]$ & 150.0 \\
\hline \hline
\end{tabular}

\section{B. Inlet total pressure profiles}

For the generation of non-uniform total pressure profiles at the inlet of the S-duct, distortion gauzes were placed at station 3 (Fig. 1), 2.55 $\mathrm{D}_{\text {in }}$ upstream the S-duct entry plane. In this work, a honeycomb screen [32] was used to produce a boundary layer-type total pressure profile [33], with a thickness of $\delta / \mathrm{D}_{\text {in }}=0.332$ (Profile A) [26] and $R e_{\delta}$ $=6.3 \times 10^{4}$, which is thicker than the nominal profile with $\delta / \mathrm{D}_{\text {in }}=0.04$ and $R e_{\delta}=4.6 \times 10^{3}$. Profile A is representative of a full scale Blended Wing Body (BWB) aircraft, which is expected to have a boundary layer near the wing-body trailing edge of about $\delta / D_{\text {in }}=0.30$ [34]. 


\section{TR-PIV experimental setup}

Time-resolved PIV measurements were performed at the Aerodynamic Interface Plane (AIP) located $0.4 \mathrm{D}_{\text {out }}$ downstream of the S-duct exit plane. A dual cavity pulsed Nd:YAG laser was used to illuminate the cross-flow plane. Di-Ethyl-Hexyl Sebacate particles, with an average diameter of approximately $1 \mu \mathrm{m}$, were used to seed the flow. PIV images were recorded by a pair of CMOS cameras in a stereo arrangement. The cameras' sensor resolution was 1280 $\mathrm{x} 800 \mathrm{px}^{2}(1 \mathrm{MP})$ with a maximum straddle frame rate of 16,600 fps. The acquisition frequency was $4 \mathrm{kHz}$, which is 10 times greater than the expected dominant flow frequency of around $S t=1.0$ [23]. Each TR-PIV dataset consists of 20,000 instantaneous velocity snapshots, which is sufficient for the convergence of the out-of-plane velocity component and the standard deviation to $0.2 \%$ and $0.4 \%$ respectively [23]. The acquired 3 -component velocity maps had a spatial resolution of $0.0153 \mathrm{D}_{\text {out }}\left(2.3 \mathrm{~mm}^{2}\right)$ across the AIP. Only the data within $r<0.95 R_{A I P}$ is used in the current analysis, in order to ensure that no spurious vectors near the domain boundaries caused by laser light reflections influence the outcomes. This results in a total of approximately 2,900 3-component velocity vectors across the AIP, which is notably richer than a conventional flow distortion measurement system that would usually provide 40 total pressure measurements across the plane [35]. A disparity correction reduced the bias errors due to misalignment between the laser light and the calibration plate. The estimated uncertainty on the velocity components was $3.3 \%$ of the area-averaged, time-averaged out-of-plane velocity at the AIP based on the method outlined by Raffel et al. [36].

\section{Unsteady swirl distortion analysis in the relative frame of reference}

Much of the previous computational and experimental work focused on the assessment of intake flow distortion for a range of geometries $[23,25]$ as well as on the effect of various flow control methods in unsteady swirl distortions $[22,35,37]$. Although some experimental studies included a rotating compression system in conjunction with either prescribed distortion or a complex intake [26], unsteady distortion measurements for such cases are very limited. In addition, most of the past work on canonical test cases such as S-ducts with circular cross sections evaluated the steady and unsteady distortion in an absolute frame of reference. This is within the context of the related industry standard metrics which are also based on an absolute frame of reference. It is also known that there is an interaction between the compression system and the distortion which arises for complex intakes [22]. Previous studies using TR-PIV provided rich measurement data sets which quantified the detailed unsteady characteristics of the absolute velocity distortion [23] for intakes in the absence of the compression system. However, the characteristic signature of the flow 
distortion perceived by the propulsion system depends also on the rotational speed of the blades through the unsteady distorted flow field. This variation is not typically evaluated with pressure probes as it is difficult to integrate the sensors into the rotating machinery. Similarly, multi-component pressure probes in a stationary frame are intrusive and have insufficient spatial resolution to indicate distortion in a nominal relative frame of reference. However, this is possible with TR-PIV data which provides synchronous 3 components of velocity across the AIP with sufficient temporal and spatial resolution. Within this context, the unsteady velocity distortion is assessed at the relative frame of reference of a notional compression system installed at the AIP. To introduce this approach, the NASA Rotor 67 fan configuration has been used [38]. The TR-PIV velocity maps are converted into the relative frame of reference of this transonic fan to quantify the unsteady characteristics in terms of the incidence angle. The NASA Rotor 67 geometry and operating point is scaled to match the rig exit diameter $\left(D_{\text {out }}=150 \mathrm{~mm}\right)$ and the rotational speed has been adjusted to maintain a constant flow coefficient between the baseline and the notional scaled down configuration for a mid-span position. This preserves the ideal relative flow angle at the nominal rotor inlet and yields an axial velocity to rotor speed ratio of $v_{z} / u=1.123$ at the blades' tip. Fig. 2 illustrates the nominal velocity triangles for the scaled rotor inlet condition. The dashed lines represent the velocities for uniform inlet flow without inlet swirl (nominal conditions), where $v_{\text {nom }}$ is the absolute velocity, $u$ is the blade speed of the fan rotor, $w_{\text {nom }}$ is the relative velocity and $\beta_{\text {nom }}$ is the relative inlet flow angle. The continuous lines represent the velocity triangle for the S-duct inlet configuration, which introduces a local swirl distortion, described with the swirl angle $\alpha$, and fluctuations in the axial velocity component $v_{z}^{\prime}$. In Fig. 2, $\alpha$ is the absolute swirl angle at the rotor inlet, $v^{\prime}$ is the absolute velocity, $u$ is the blade speed, $w^{\prime}$ is the relative velocity, $v_{z}^{\prime}$ and $v_{\theta}{ }^{\prime}$ are the axial and circumferential components of the absolute velocity $v^{\prime}$ respectively. 


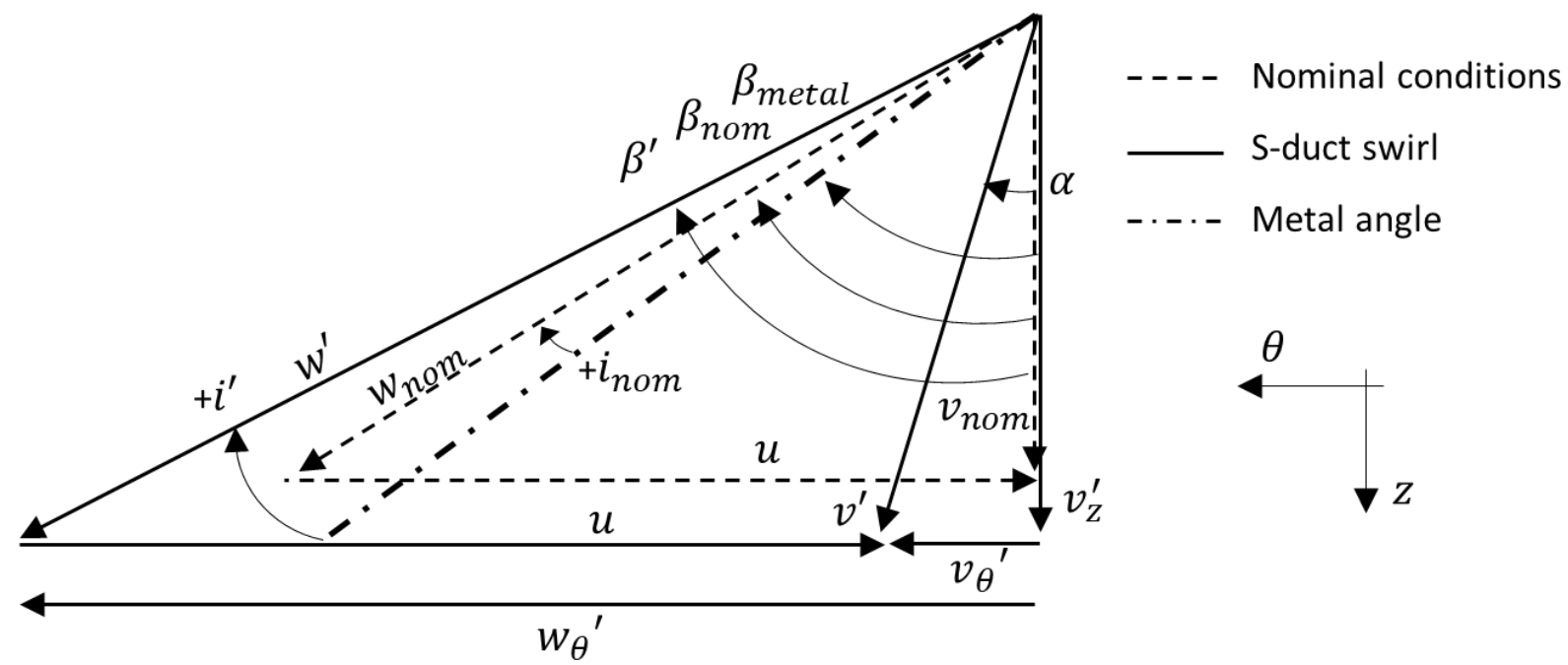

Fig. 2: Velocity triangles at the rotor leading edge for nominal conditions (dashed line) and in case of swirl distortion (continuous line).

The relative flow angle in case of swirl distortion is defined as:

$$
\beta^{\prime}=\tan ^{-1}\left(w_{\theta}{ }^{\prime} / v_{z}{ }^{\prime}\right)
$$

where $w_{\theta}{ }^{\prime}$ is the relative swirl velocity and $v_{z}$ ' is the axial velocity. The incidence angle on the rotor blades for nominal inlet conditions (dashed line Fig. 2) is defined as the difference between the relative flow angle $\beta_{\text {nom }}$ and the metal angle $\beta_{\text {metal }}$ at the rotor inlet (dash-dot line in Fig. 2):

$$
i_{\text {nom }}=\beta_{\text {nom }}-\beta_{\text {metal }}
$$

Similarly, the incidence angle on the rotor blades for the S-duct inlet flow configuration (continuous line Fig. 2) is defined as the difference between the relative flow angle $\beta^{\prime}$ and the metal angle at the rotor inlet $\beta_{\text {metal }}$ (dash-dot line in Fig. 2):

$$
i^{\prime}=\beta^{\prime}-\beta_{\text {metal }}
$$

The perturbations of the incidence angle due to the local swirl distortion with reference to the nominal incidence with uniform axial flow can be expressed as (Fig. 2):

$$
\Delta i=i^{\prime}-i_{\text {nom }}
$$

In the subsequent sections, the analysis will focus on the incidence angle perturbations $(\Delta i)$ with reference to the nominal R67 incidence. 


\section{E. Exposure time, intensity and probability of distortion events}

The unsteady flow distortion is characterized by temporal variations across a range of frequencies and spatial variations. For example, a nominal compressor rotor will be exposed to different levels of flow distortion at different azimuthal positions, all of which also vary in time. Therefore, the levels of unsteadiness onto the nominal rotor blade leading edge will depend on both the blade position (which in turn depends on its speed and original position), and the severity of the local unsteady flow distortion, which is dictated by its time and spatial characteristics. The distortion for a nominal rotor blade as it rotates around the annulus will depend on the nominal starting position as well as the nominal starting time relative to the unsteady distortions. Therefore, it is necessary to evaluate the distortion for each potential starting azimuthal position of each rotor blade relative to the full unsteady flow measurements which encompass the equivalent of several hundred rotations of the rotor. Clearly, for each rotor, the unsteady distortion will also vary in the spanwise direction. The nature of the TR-PIV data which provides synchronous three component velocity fields across the AIP at a sufficient temporal resolution enables all of these factors to be assessed for the first time. Conventional unsteady total pressure measurements with fixed rakes cannot provide this information. Similarly, unsteady data from typical five-hole probe devices do not provide the required synchronous spatial resolution to enable such an evaluation. Within this context, for a notional, scaled down R67 configuration with 22 blades, the blade speed would be $18,684 \mathrm{rpm}$, with a $1 \mathrm{EO}$ frequency of about $311 \mathrm{~Hz}$. The acquisition frequency of the TR-PIV was $f_{s}=4$ $\mathrm{kHz}$; therefore, the time step between two successive snapshots was equal to $\Delta t_{P I V}=1 / f_{s}=0.25 \mathrm{~ms}$. This results in approximately 1,550 notional rotor revolutions during the total acquisition time of the 20,000 velocity fields of the TR-PIV dataset. Therefore, for a notional rotor blade 0 positioned at a reference angle $\theta_{b 0}=0^{\circ}$ at time $t_{0}=0$, at the subsequent time $t_{1}\left(t_{0}+\Delta t_{P I V}\right)=0.25 \mathrm{~ms}$, the blade will have moved across a sector to the azimuthal position:

$$
\theta_{b 0}\left(t_{1}\right)=\theta_{b 0}\left(t_{0}\right)+\omega\left(t_{1}-t_{0}\right) \sim 28.0 \mathrm{deg}
$$

where $\omega$ is the rotor angular speed in rad/s. The rotor speed is kept constant, therefore at snapshot $t_{2}=2 \Delta t_{P I V}=$ $0.5 \mathrm{~ms}$, blade 0 will be at $\theta_{b 0}\left(t_{2}\right)=56.0 \mathrm{deg}$. This is illustrated in Fig. 3 which shows the blade rotation for 3 PIV snapshots where the incidence varies both in space and time. The time is normalized by passing time of the rotor blades $\left(t_{b p}\right)$. The incidence angle on a specific rotor blade depends on the time at which the incidence is measured and the circumferential position of the notional blade at the same time instant. This time-coupling between the rotor rotation and the TR-PIV dataset considers both sources of variability. 


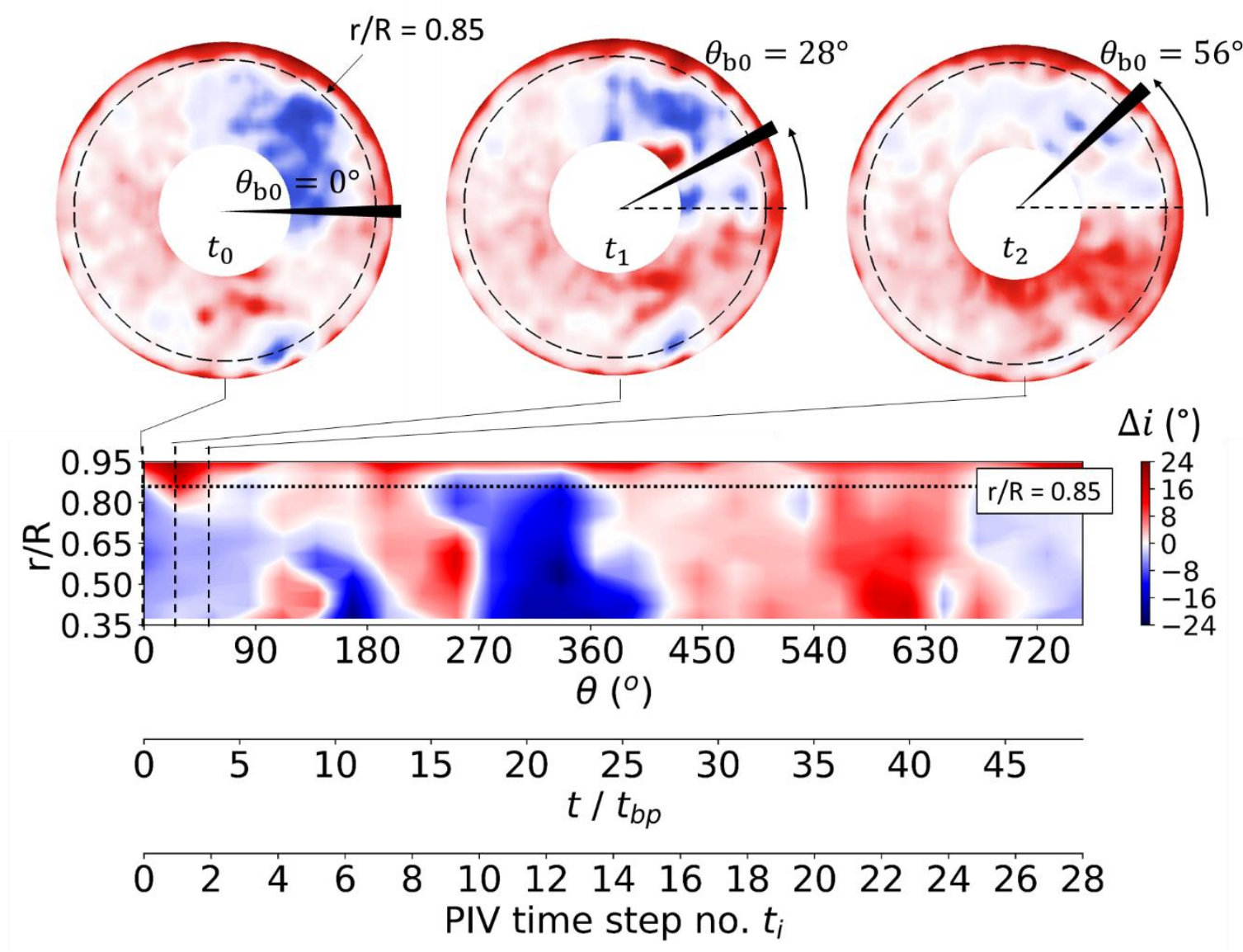

Fig. 3: Time coupling between the TR-PIV dataset snapshots and the rotation of the compressor rotor.

The incidence angle was assessed for different radial positions. Analogous with the established swirl distortion descriptor definitions [2], the perturbations of the incidence angle $(\Delta i)$ with reference to the nominal R67 inlet with no inlet swirl and uniform flow conditions (Fig.2) are classified as distortion events. Each distortion event is described by two properties: the exposure time and a magnitude value (Fig. 4). The exposure time $\left(t_{e}\right)$ is defined as the period of time between two changes of sign of the incidence angle perturbation $(\Delta i)$ and it is normalized against the blade passing time $\left(t_{b p}\right)$. Thus, it represents the effective duration of the distortion event in the relative frame of reference. It represents also the changes in the approaching flow conditions for an individual rotor blade during the rotation relative to the start of the unsteady data. The magnitude of each distortion event is quantified with the mean $\left(\langle\Delta i\rangle_{e}\right)$ value of incidence angle. 


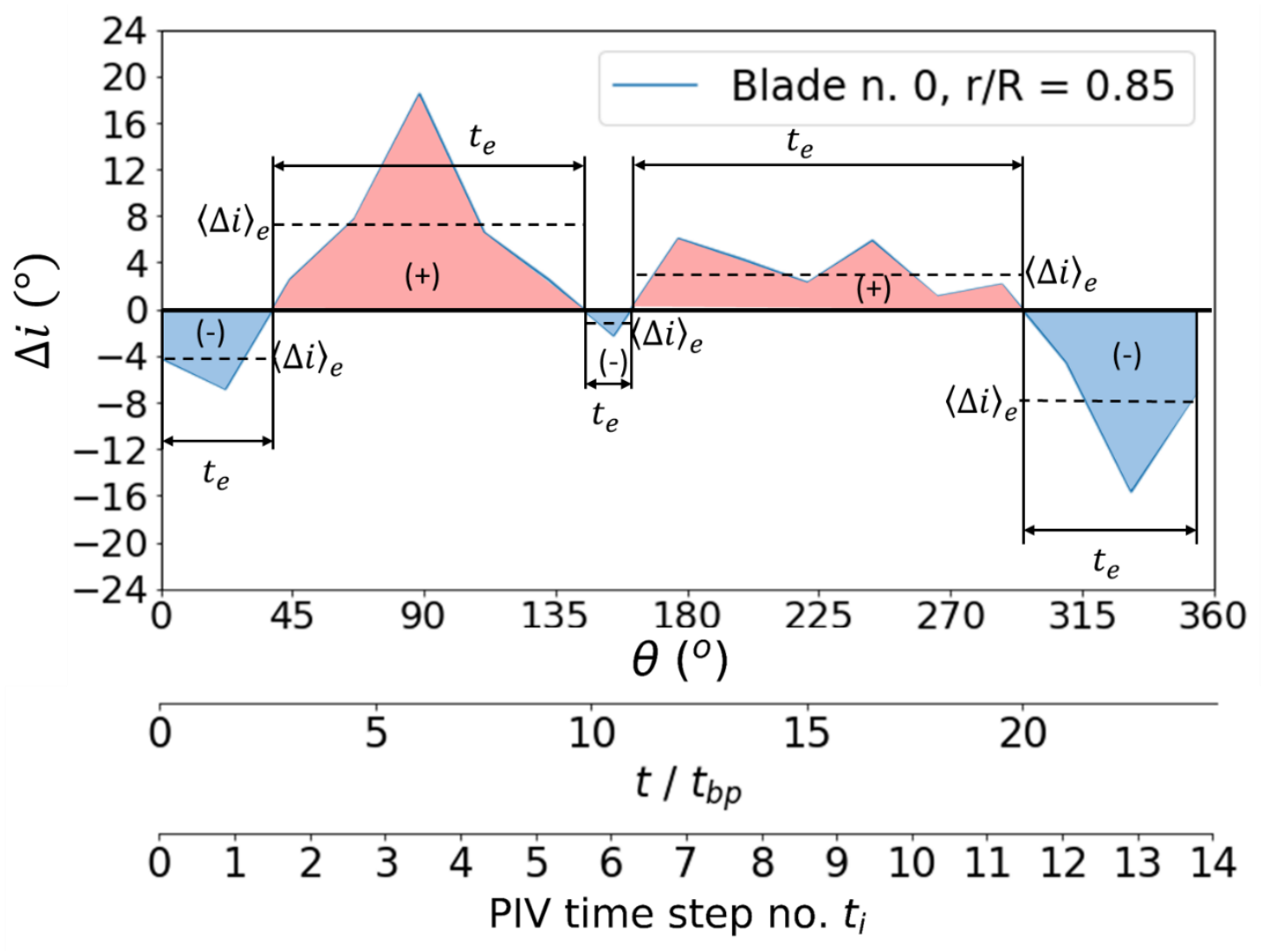

Fig. 4: Classification of the distortion events by the change in sign of the incidence angle perturbation.

A separate approach is proposed to identify extreme distortion events. Most of the distortion events identified with the change of sign of the mean incidence are within a range of values that could potentially be tolerated by the propulsion system. However, for unsteady flow distortion the mean values are not the primary interest. The most important, and the least understood, are the peak distortion events associated with a significant value of incidence that could generate separation and trigger stall inception on the compressor blades. Therefore, a different approach is proposed in order to highlight these extreme events and to consider only the ones which deviate substantially from the standard fluctuations. Thus, the events are classified as peak events if the mean change in incidence $(\Delta i)$ exceeds the sum of the mean value within the event $\left(\langle\Delta i\rangle_{e}\right)$ and one standard deviation of the fluctuations (full signal):

$$
\exists \Delta i_{\text {peak }} \leftrightarrow \Delta i_{\text {peak }}>\langle\Delta i\rangle_{e}+\operatorname{std}\left(i^{\prime}\right)
$$

When this condition is valid, each peak event is classified again by two quantities: the exposure time $\left(t_{e}\right)$ and the magnitude value (Fig. 5). In this case, the exposure time is defined as the time in which the change in incidence is above $50 \%$ peak height, while the magnitude value is the maximum incidence $(\Delta i)$ of the peak event. 


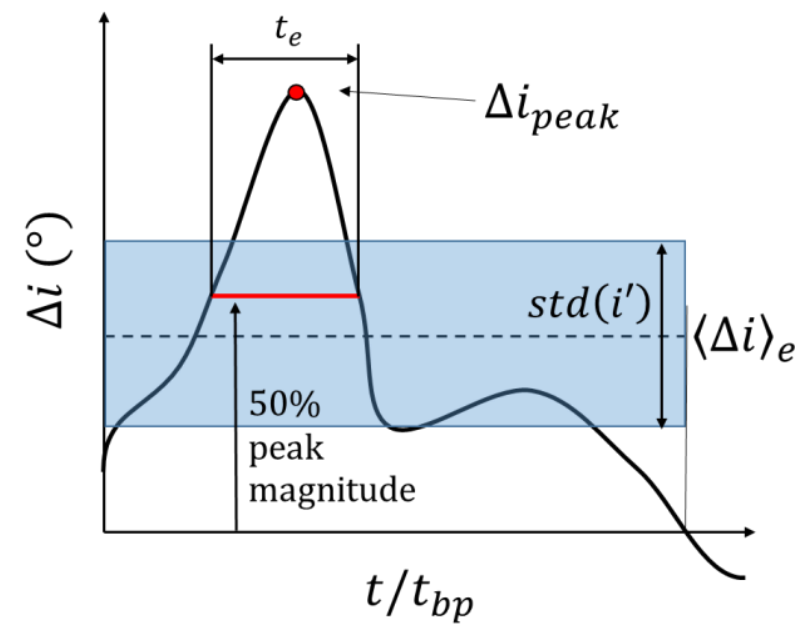

Fig. 5: Identification of the peak events which exceed mean value and one standard deviation.

Although the assessment of the incidence perturbations characteristics such as magnitude and spanwise distribution has been highlighted for one blade (Fig. 3), it also applies for all the rotor blades of the notional R67. The position of each blade is defined at any instant in time as $\theta_{b n}\left(t_{i}\right)$, where $t_{i}$ is a time instant of the TR-PIV dataset and $n$ identifies a generic blade. Thus, the change in incidence can be evaluated for each blade as a function of:

$$
\Delta i_{b n, r}=\Delta i\left(\theta_{b n}, r\right)
$$

where $\theta_{b n}\left(t_{i}\right)$ is the circumferential position of the $n$-th blade in the velocity field, $r$ is the radial coordinate and $n=$ $\left[0, N_{\text {blades }}\right]$ with $N_{\text {blades }}$ the total number of blades. This increases the number of samples in the dataset by a factor equal to the number of the notional blades. For example, in this work, the number of samples of incidence angles considering one blade $\theta_{b 0}$ is 20,000 , which is the number of instantaneous velocity snapshots in the TR-PIV dataset. Considering all the 22 rotor blades [38], the total samples increases to 440,000 .

However, for a given blade starting from a certain initial circumferential location (see $\theta_{b 0}$ in Fig 6a), the analysis described so far would not account for any distorted region located within a sector shorter than the minimum sector of $28.0^{\circ}$ as defined by the TR-PIV acquisition frequency and the notional blade speed. For example, if a distortion event occurs within the extent of a blade pitch $\left(\Delta \theta_{b}=16.3^{\circ}\right)$ in snapshot 1 (Fig. 6a), it would not be captured by this analysis of blade "0", even if it were measured by the TR-PIV. More importantly, it is possible that this event would not be captured in the successive snapshot either. This could happen if the distortion region remains in the same location in the next time step (Fig. 6b) and does not coincide with any of the angular positions of the blades. Alternatively, it could happen that the distortion region moves circumferentially with the same speed of the rotor and 
therefore, it will be located again in the non-evaluated part of the blade pitch region (Fig. 6c). Or, finally, it could happen that the unsteady distorted region which is in-between the blade pitch at snapshot 1 disappears by snapshot 2 (Fig. 6d). In all these cases, the distorted region is not captured by this method.

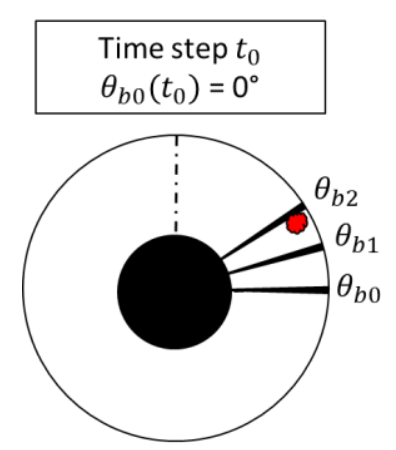

a)

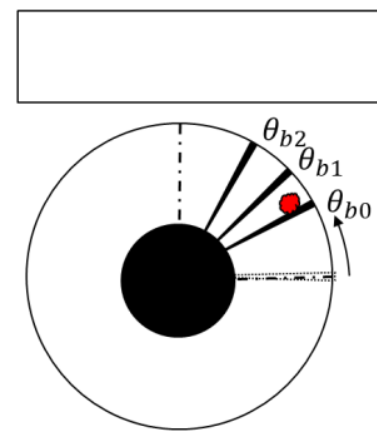

b)
Distortion event

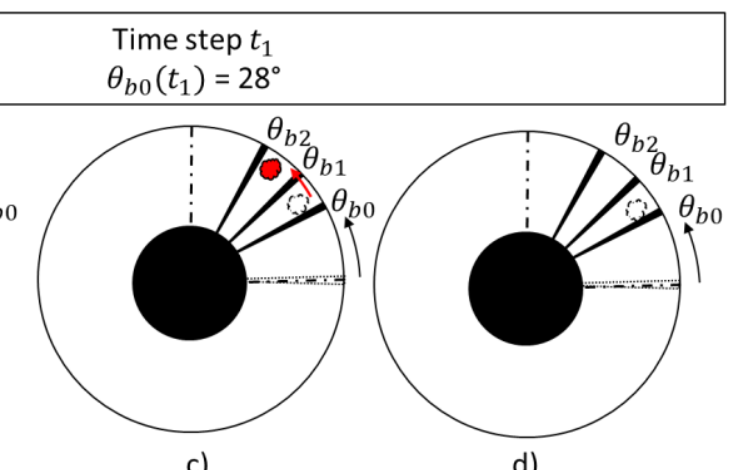

c)

d)

Fig. 6: Distortion events within the blade pitch and between successive time steps.

However, the distorted region of Fig. $6 \mathrm{a}$ is captured by the TR-PIV measurements. The nominal position of the blade 0 at the start of the PIV acquisition is defined arbitrarily and therefore an angular offset to the starting position of the rotor could align at least one of the blades with the distortion region (Fig. 7). Therefore, to get a more statistically representative dataset of the flow distortion from the perspective of a full rotor set, the analysis was extended to include a range of angular offset angles within $\Delta \theta_{\text {pitch }}=16.3^{\circ}$. The number of the clocked positions has been defined by the coarsest angular grid resolution of the TR-PIV acquisition at the blades tip ( $2.3 \times 2.3 \mathrm{~mm})$, which corresponds to an angle of $\Delta \theta_{\text {clocking }}=1.75^{\circ}$. This equates to $\Delta \theta_{\text {pitch }} / \Delta \theta_{\text {clocking }} \approx 9$ positions. With this construction, the set of clocked Rotor 67 ( 22 blades) is equivalent to 206 blade instances and the total number of samples of incidence angle at each radial position $\theta_{b n}\left(t_{i}\right)$ is about $4.1 \times 10^{6}$. This approach improves the representative sampling and statistics, firstly because of the different starting position of the rotor at the beginning of the TR-PIV acquisition, and secondly because of the increased resolution across a blade pitch. However, it should be noted that this approach does not increase the sampling frequency of the unsteady incidence signals of the blades rotation. Although the starting position of the blade can be offset by $\Delta \theta_{\text {clocking }}$, the rotation of the blade between adjacent PIV time steps will remain $\Delta \theta_{b}$ and it will be independent of the clocked position. 

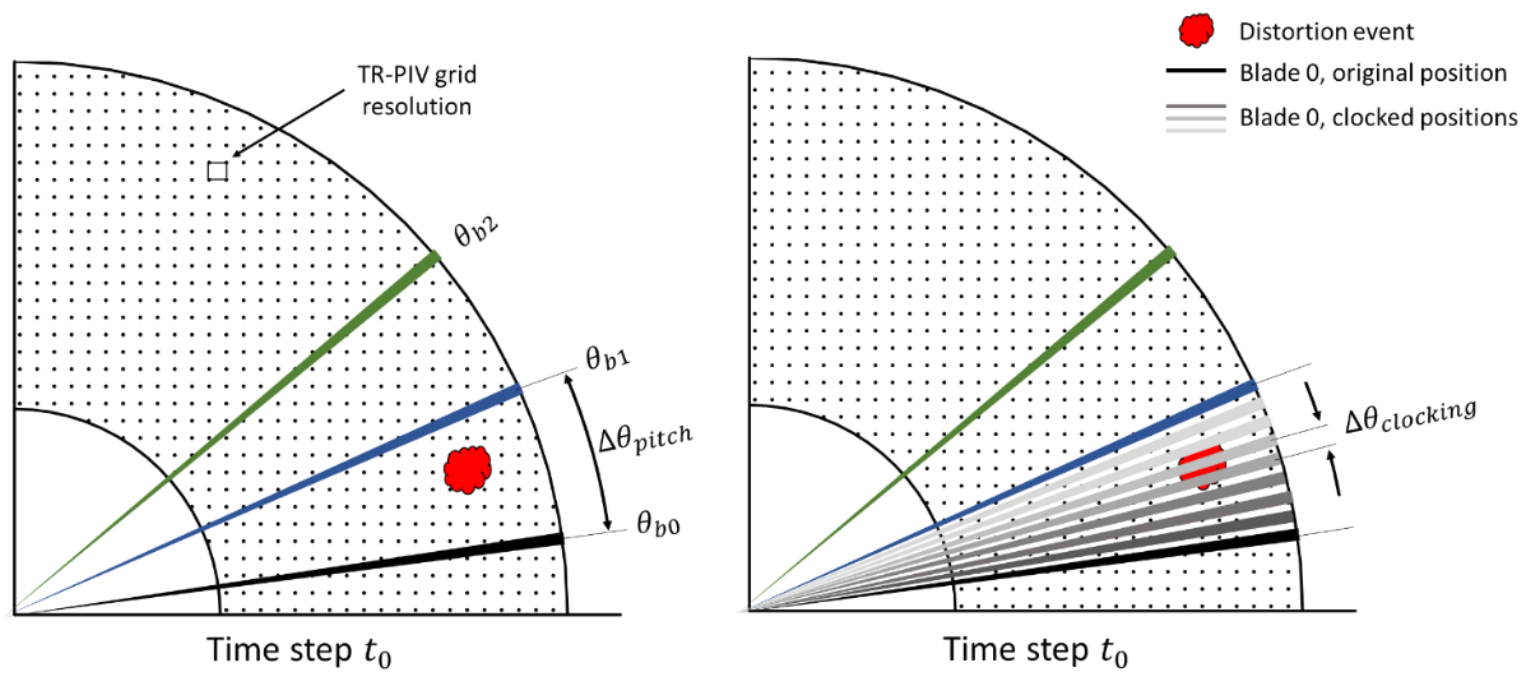

Fig. 7: Clocking positions of the rotor to improve the distortion events detection.

Nevertheless, this work has some limitations. Firstly, it considers an envisaged relative frame of reference for Rotor 67. The data is captured at the outlet of the S-duct intake without the rotor geometry placed in the section. Thus, the current data do not include the influence of the fan on the intake distortion characteristics. In addition, only the data in the hub-to-tip annular sector $\left(r>R_{\text {spinner }}\right.$ with $\left.R_{\text {spinner }}=0.372\right)$ is considered to compute the incidence angles. In spite of these limitations, the aim of the work is to establish a new analysis approach for the assessment of the unsteady flow distortion more closely related to the aerodynamic characteristics of a propulsion system that could be adopted for a range of experimental and computational studies.

\section{Results}

In the next sections, the novel methodology is applied to assess the distortion events generated by the inherent flow distortion of the S-duct intake. The reported levels of swirl distortion are relatively large since the likely suppression effect of the fan and hub are not included. However, the main intent of the work is to analyze combined unsteady temporal and spatial swirl fluctuations as it would be presented to rotating fan blades. With this approach, both the magnitude of the distortion event ( $\Delta i_{\text {mean }}$ and $\Delta i_{\text {peak }}$, Section II.E) and the period of time during which the blade is exposed to the distorted event are of interest and are considered simultaneously. Thus, the focus is directed to the probabilistic distribution maps in which the correlation between magnitude and blade exposure time among the population of distortion events is projected. Events with relatively modest magnitude which last in the order of 1 or 
more revolutions are assessed with the average incidence distribution metric $\Delta i_{\text {mean }}$ (Section II.E). More discrete events relative to bulk flow fluctuations with a duration in the order of the blade passing time are assessed with the peak change of incidence metric $\Delta i_{\text {peak }}($ Section II.E). Of particular interest are events with peak positive incidence, since these may trigger the spike-type stall inception [15]. As Cousins [3] demonstrated, the compressor response to the inlet distortion is linked with the exposure time to the distortion. Thus, the duration of these peak distortion events is also of notable importance. For an axial compressor, events with duration between 4-5 blade passing time may cause instabilities and this method can reveal the maximum magnitude of distortion events within this duration range. To give an overview of a broader range of distorted conditions, the method is applied to uniform inlet conditions as well as different S-duct inlet configurations. This aims to demonstrate that this method can be used to discern differences for a range of unsteady flow distortion flow fields that may not be evident using conventional methods. Of particular interest is the signature of the flow distortion in terms of the level of the peak distortion as a function of blade exposure time for the various inlet conditions.

\section{A. Flow field in the relative frame of reference}

The basic flow topology is highlighted by the time-averaged velocities at the AIP and the nominal incidence angles (Fig. 8). The velocities are normalized by the time-averaged, area-averaged out-of-plane velocity at the AIP $\left(\overline{\left\langle v_{z}^{\prime}\right\rangle}\right)$. The central region of the AIP has been cropped for $r<R_{\text {spinner }}$ to focus on the nominal blade span region of R67 only [38]. The out-of-plane velocity component $\left(v_{z}^{\prime}\right.$, Fig. 8a) shows the typical loss region at the bottom of the section identified in the work of Zachos et al. [18] with a low-bandwidth PIV data on the same high-offset S-duct. The maximum values of the time-averaged out-of-plane velocity component $\left(v_{z}^{\prime}\right.$, Fig. $\left.8 \mathrm{a}\right)$ are in good agreement with the range of velocities previously identified by Gil-Prieto et al. [20] for an inlet Mach number of 0.27. The tangential velocity $\left(v_{\theta}^{\prime}\right.$, Fig. $\left.8 \mathrm{~b}\right)$ primarily highlights the vertical movement of the secondary flows that are generated within the S-duct. The streamlines highlight the pair of contra-rotating Dean vortices [39] typical of curved ducts. The tangential velocity is one order of magnitude smaller than the out-of-plane component. Thus, the time-averaged swirl angle $\alpha$ associated with the tangential component of the velocity $v_{\theta}^{\prime}$ (Fig. 8b) ranges between $\pm 8^{\circ}$. The slight asymmetry which is visible in the streamwise velocity $\left(v_{z}^{\prime}\right.$, Fig. $\left.8 \mathrm{a}\right)$ and tangential velocity $\left(v_{\theta}^{\prime}\right.$, Fig. 8b) are attributed to the measurement uncertainty as well as non-uniformities in the intake. 
The flow field in the rotor relative frame of reference is now considered. The relative tangential velocity $\left(w_{\theta}^{\prime}\right.$, Fig. 8c) has a positive bias in the relative time-averaged tangential velocity, since the rotational speed of the rotor $u$ is one order of magnitude greater than the flow absolute tangential velocity $v_{\theta}^{\prime}$. The gradient of the relative tangential velocity $\left(w_{\theta}^{\prime}\right.$, Fig. 8c) reflects the natural radial gradient of the rotational speed of the rotor. The contra-rotating pattern of the tangential velocity $v_{\theta}^{\prime}$ only marginally influences the time-averaged relative tangential velocity but is sufficient to generate some asymmetry in the relative velocity component distribution $\left(w_{\theta}^{\prime}\right.$, Fig. 8c) due to the effect of the rotational speed and direction. The incidence angle $\left(i^{\prime}\right.$, Fig. 8d) has been computed between the relative flow angle $\beta^{\prime}$ and the nominal metal angle of R67 ( $\beta_{\text {metal }}$, Fig. 2). The time-averaged distribution shows a zone of negative incidence $\left(\left\langle i^{\prime}\right\rangle\right)$ in the right part of the domain, and a region with high values of about $+5^{\circ}$ in the lower half of the domain close to the hub. The first zone corresponds to an area with a relatively high axial velocity component $\left(v_{z}^{\prime}\right.$, Fig. 8 a) and a modest relative tangential velocity $\left(w_{\theta}^{\prime}\right.$, Fig. $\left.8 c\right)$, which produces a reduction in the relative flow angle $\left(\beta^{\prime}\right.$, Fig. 1) and a consequent negative incidence $\left(\left\langle i^{\prime}\right\rangle\right)$. The second zone corresponds to an area with an axial component $\left(v_{z}^{\prime}\right.$, Fig. 8a) that is lower than the relative tangential velocity $\left(w_{\theta}^{\prime}\right.$, Fig. 8c). This produces an increase of the relative flow angle $\left(\beta^{\prime}\right.$, Fig. 1) and a consequent positive incidence $\left(\left\langle i^{\prime}\right\rangle\right)$.

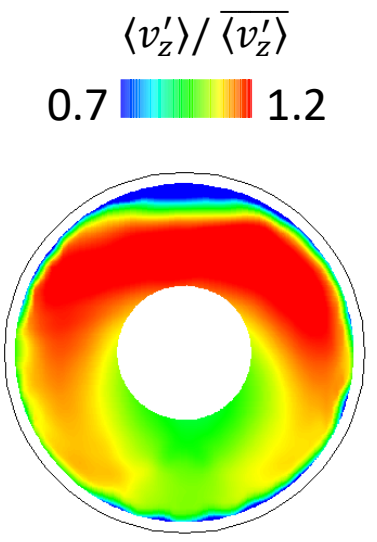

a)

\section{$\left\langle v_{\theta}^{\prime}\right\rangle / \overline{\left\langle v_{z}^{\prime}\right\rangle}$}
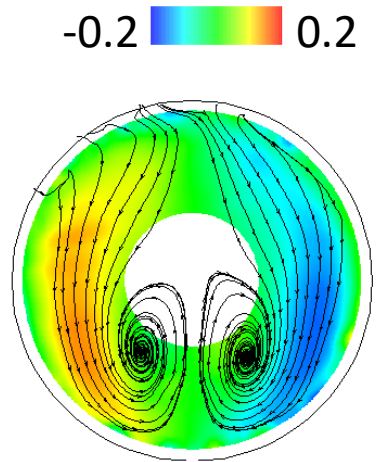

b)
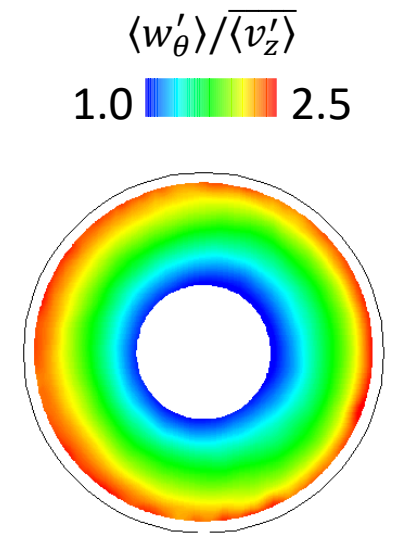

c) $\left\langle i^{\prime}\right\rangle\left(^{\circ}\right)$
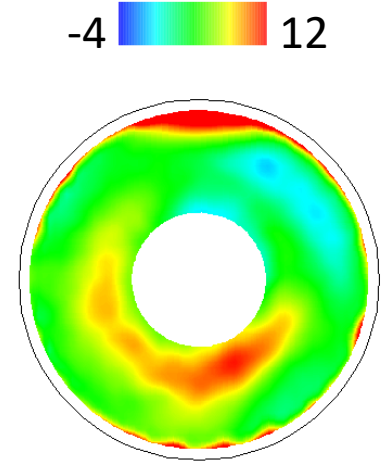

d)

Fig. 8: Time-averaged flow field with nominal inlet boundary layer $\left(\delta / D_{\text {in }}=0.04\right)$.

Of particular interest is the time history of the incidence perturbations $(\Delta i)$ across the AIP. During one revolution, the rotor blades are exposed to several changes of incidence that could influence rotor operability, vibrational response and promote blade stall $[26,10]$. Recent studies showed that vortex-induced swirl events could cause a substantial reduction in the compressor total pressure ratio [40]. This highlights the possible flow compatibility issues between 
the curved intake and the rotor compressor. The radial positions that are subject to off-design conditions are visible in the time history of the blade incidence angle $(\Delta i)$ (Fig. 9). Only 2 out of the overall 1,550 revolutions are shown in the time history sample for 1 blade. Despite the relatively short time period, large fluctuations of incidence $(\Delta i)$ of up to $\pm 24^{\circ}$ are indicated. Different events are highlighted in Fig. 9 at times a), b) and c). At time a), the incidence angle is negative across the entire span, therefore the blade loading is likely decreased in comparison to the design point. At time b), the incidence angle is close to $+5^{\circ}$ along the span; so, the blade loading is expected to increase. At the time c), the incidence angle is positive near the hub while it is negative near the tip; so, the blade is experiencing a nonuniform blade loading distribution. This situation is similar to the result of Giuliani and Chen's simulation [26] in which a blade experienced uneven hub-to-tip incidence and total pressure distribution during the exposure to a contrarotating pair of S-duct vortices. These conditions were depicted as possible causes of dynamic blade stall due to the high values of incidence angle and the pressure difference between hub and tip locations. This result strengthens the rationale of the unsteady incidence angle assessment for the identification of stall inception cells and uneven blade loading.

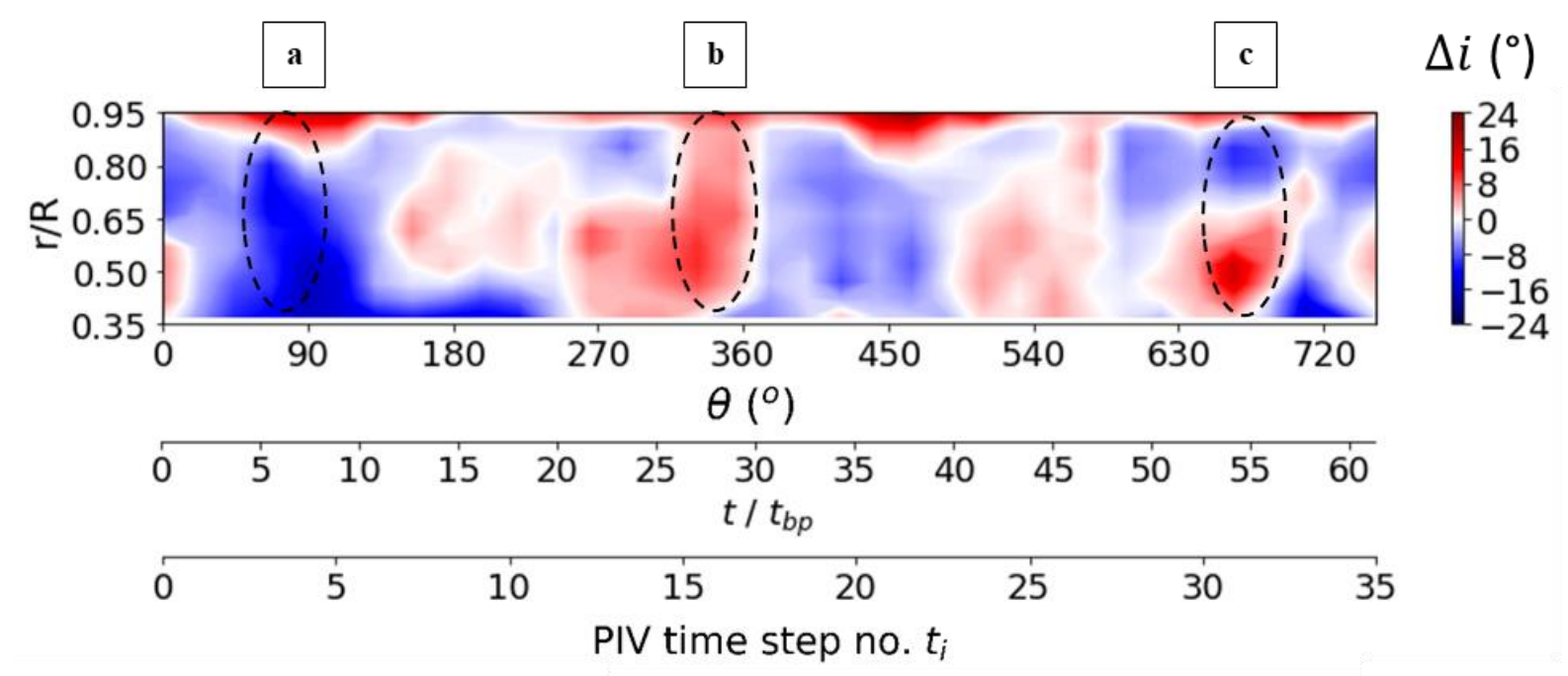

Fig. 9: Incidence angle perturbations for 1 blade over 2 revolutions in the distorted flow. 


\section{B. Identification of distortion events}

The tracking of the incidence angle perturbations $(\Delta i)$ is shown at the tip $(\mathrm{r} / \mathrm{R}=0.85)$ and hub $(\mathrm{r} / \mathrm{R}=0.51)$ radial positions using the scheme described in Fig. 3 for the identification of distortion events (Fig. 4). Each event is classified with two variables: the exposure time $\left(t_{e}\right)$, which is the time the event persists on the blade during the rotation $\left(\theta_{b n}\right)$, and the magnitude of the change in incidence, which can be either the mean $\left(\langle\Delta i\rangle_{e}\right)$ or the peak value $\left(\Delta i_{\text {peak }}\right)$ within the exposure time (Fig. 4). This identification allows to represent the data in a Cartesian plane in which the exposure time is on the horizontal axis and the mean or peak value are in the vertical axis (Fig. 10). The resolution of the exposure time is set by the rotation angle between two successive TR-PIV snapshots $\left(\Delta \theta_{b}=28.0^{\circ}\right)$. This rotation angle sets also the minimum exposure time of the detected events, since it is not possible to detect any events shorter than the TR-PIV sampling frequency. The exposure time $\left(t_{e}\right)$ is normalized by the blade passing time $\left(t_{b p}=2 \pi / \omega N_{\text {blades }}\right)$, which is consistent with the conventional way of reporting the range of critical frequencies for axial compressors [3]. For typical transonic compressors the blade passing frequencies is approximately twice the reduced frequency based on rotor chord and average convective velocity form leading to trailing edge.

The mean and peak value of the incidence angle perturbations is interpolated in grid steps of $0.5^{\circ}$. The distortion events that are identified in the TR-PIV dataset populate the grid depending on the magnitude and the exposure sector. In the exposure maps, the probability of the events is computed by enumerating all the events characterized by the same magnitude and exposure sector. The value is then normalized by the total number of distortion events detected at the blade tip $(\mathrm{r} / \mathrm{R}=0.85)$ to compute the probability, which is then reported in a logarithmic scale. The boundary between event detection / no detection in the exposure-magnitude plane is indicated with the dashed line (Fig. 10). The selected approach considered 1,550 rotor revolutions, all R67 blades and rotor offset positions (see section II.E) and evaluated a total of 4.1 million spatial and temporal positions assumed by the rotor blades. Only two spanwise locations have been surveyed in representative locations at the blade tip and hub. The unsteady signals at these locations analyzed with the distortion events identification approach (Fig. 4) revealed 1.3 million distortion events at the tip position $(\mathrm{r} / \mathrm{R}=0.85)$ and 0.9 million at the hub position $(\mathrm{r} / \mathrm{R}=0.51)$, of which about 150,000 are peak distortion events (as defined in Section II.E).

The example measurement dataset used in this study is for a relatively simple S-duct in the absence of a compressor. Consequently, the reported levels of swirl distortion are relatively large as the possible suppression effect 
from the rotor and centrebody are not included. However, the basis of the new analysis method is valid and can be useful as a new approach for subsequent studies where the rotor and centrebody are included.

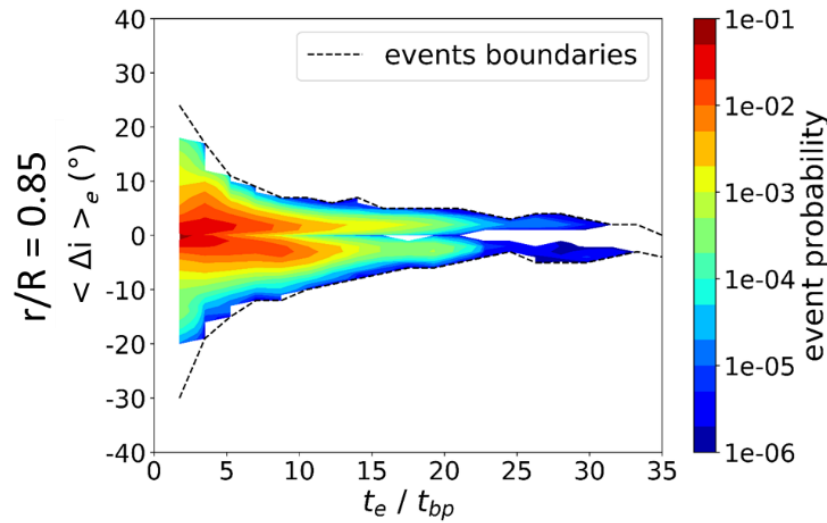

a)

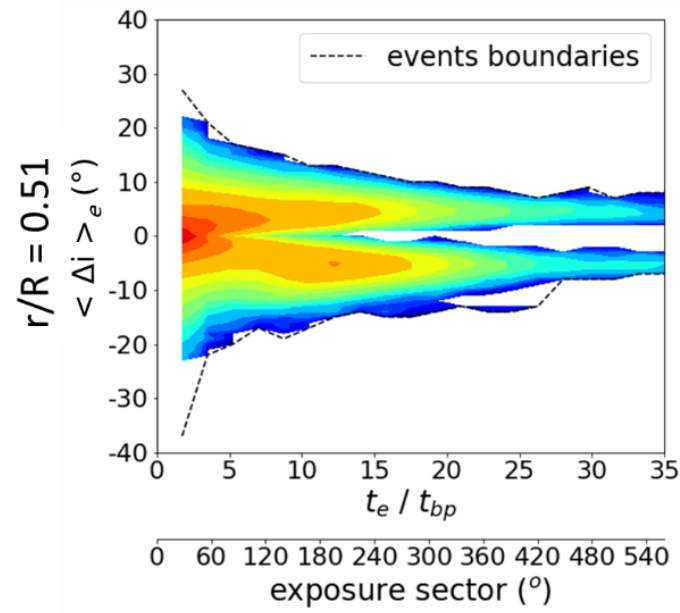

c)

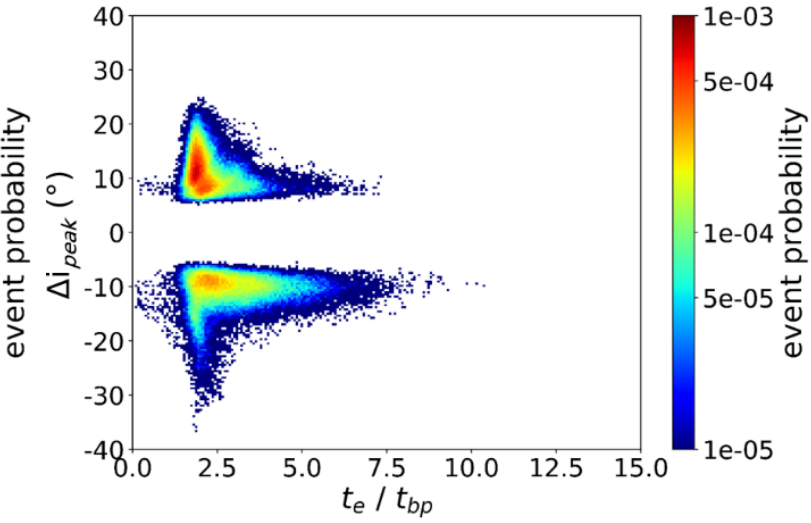

b)

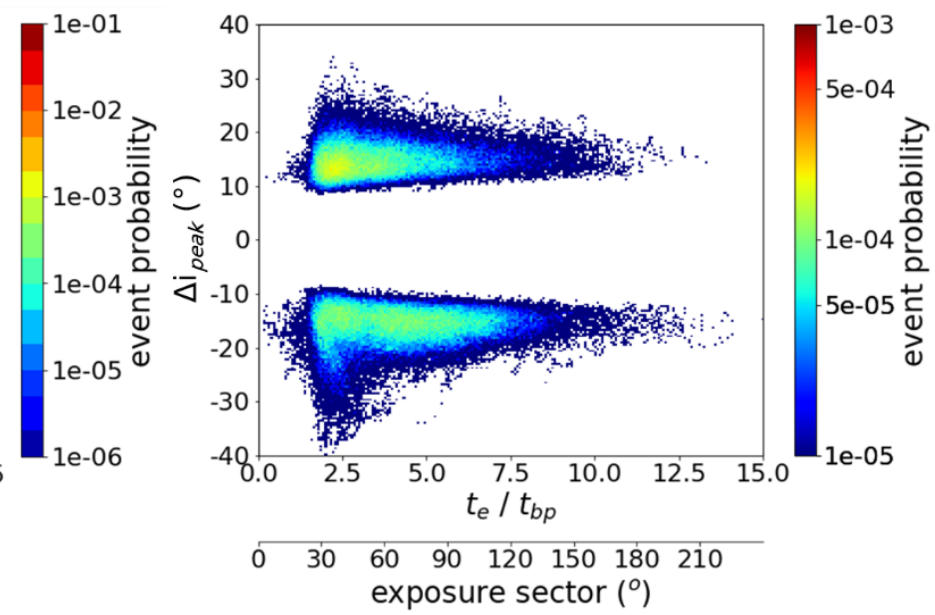

d)

Fig. 10: Probability maps for distortion events with nominal inlet boundary layer $\left(\delta / D_{\text {in }}=0.04\right)$ for hub $(r / R=$ $0.51)$ and tip $(r / R=0.85)$ positions.

For a position close to the tip $\left(\mathrm{r} / \mathrm{R}=0.85\right.$, Fig. 10a), the mean incidence angle perturbations $\left(\langle\Delta i\rangle_{e}\right)$ cover a range between $-30^{\circ}$ and $+25^{\circ}$. The exposure sector $\left(t_{e}\right)$ of the distortion events ranges from the minimum time step between two successive PIV acquisitions $\Delta \theta_{b}=28.0^{\circ}$ and a maximum duration equivalent to about 1.5 rotor revolutions (Fig. 10a). The probability is relatively balanced between positive and negative events. However, there is a higher probability of relatively short events with positive incidence. For example, between 0 and 5 blades passing, positive events are more probable than the negative ones, although they have a similar maximum magnitude. This can relate to the flow separation caused by the secondary bend of the S-duct intake. This is visible near the top duct boundary in 
the out-of-plane velocity component (Fig. 8a) and it is reflected in the time-averaged incidence angle distribution (Fig. $8 d)$. In general, events with a longer exposure have a comparatively modest change of incidence $\left(\langle\Delta i\rangle_{e}\right)$. For example, events with an exposure sector of $360^{\circ}$ have a maximum incidence perturbation between $+5^{\circ}$ and $-5^{\circ}$ (Fig. 10a). This is also reflected in the time-averaged incidence distribution (Fig. 8d), which shows that for a position close to the blades tip $(r / R=0.85)$ the nominal incidence is about $+4^{\circ}$ and it does not show large circumferential perturbations.

At the hub region $(\mathrm{r} / \mathrm{R}=0.51)$, the mean value of the distortion events $\left(\langle\Delta i\rangle_{e}\right)$ ranges between $-38^{\circ}$ and $+28^{\circ}$ (Fig. 10c), which is a wider range compared with the ones identified at the blades tip (Fig. 10a). Moreover, the mean value incidence angle perturbations $\left(\langle\Delta i\rangle_{e}\right)$ are $\pm 5^{\circ}$ wider on average across all the range of exposure sectors. The maximum exposure sector $\left(t_{e}\right)$ for distortion events increases also from about 1.5 revolutions to about 2 revolutions when comparing the events located at the tip $(r / R=0.85$, Fig. 10a) and at the hub $(r / R=0.51$, Fig. 10c) respectively. The distribution of probability of distortion events is similar for hub and tip positions, but, at the hub ( $r / R=0.51$, Fig. 10c) there is an increased probability of negative incidence angle perturbations. For example, for an exposure sector of $t_{e}$ $=13$ blade passing times, events which have approximately a mean change in incidence angle $\left(\langle\Delta i\rangle_{e}\right)$ of approximately $-5^{\circ}$ have a probability of $1 / 100$ at the hub $(\mathrm{r} / \mathrm{R}=0.51)$, while they have a probability of $1 / 1000$ at the tip.

Of particular interest for the engine stability assessment are the peak events $\left(\Delta i_{\text {peak }}\right)$, which could trigger the spiketype stall inception [15] (Fig. 10b, d). This work reveals extreme events which are an order of magnitude greater than time-averaged values (see Fig. 8) and which have exposure sectors between 30 and $90^{\circ}$. For example, at the tip (r/R $=0.85$, Fig. 10a), there are events with maximum mean incidence perturbations $\left(\langle\Delta i\rangle_{e}\right)$ of about $+10^{\circ}$ and exposure time about $5 t_{b p}$. Within events of the same duration $\left(5 t_{b p}\right)$, peak events $\left(\Delta i_{p e a k}\right)$ with change of incidence up to $+15^{\circ}$ were recorded (Fig. 10b). Even higher peak incidence perturbations up to $+26^{\circ}$ and $-37^{\circ}$ were identified for exposure sectors between 2 and $2.5 t_{b p}$. There is also evidence of peak events which are modest in magnitude but they have long exposure sectors. For example, at the tip ( $\mathrm{r} / \mathrm{R}=0.85$, Fig. $10 \mathrm{~b})$, there is evidence of positive peak incidence events with exposure time up to $8 t_{b p}$ and negative peak incidence events with exposure time up to $11 t_{b p}$. In this configuration, this corresponds to exposure sectors of about $110^{\circ}$ and $180^{\circ}$, respectively.

The analysis revealed substantial variation in the radial distribution of peak distortion events along a single notional rotor blade. At the tip region $(\mathrm{r} / \mathrm{R}=0.85)$, the positive peak events have a maximum exposure time of about $7 t_{b p}$ (Fig. 10b), while at the hub $(r / R=0.51)$ the positive peak events have exposure time also up to about $15 t_{b p}$ (Fig. 10d), which corresponds to more than one full revolution. The maximum magnitude $\left(\Delta i_{\text {peak }}\right)$ of the peak events increases 
from $+26^{\circ}$ to about $+35^{\circ}$ when moving from a tip position to a hub position. On the other hand, the distribution of the peak negative events in terms of maximum magnitude and exposure remains unchanged, with maximum negative change in incidence of about $-40^{\circ}$. The maximum exposure time of peak events increases slightly from about $11 t_{b p}$ at the tip (Fig. 10b) to about $15 t_{b p}$ at the hub (Fig. 10d). Similar observation can be done for the distribution of mean incidence events at the two radial positions (Fig. 10a, c).

The positive incidence events at the blades tip are likely to cause separation on the blades and promote spike-stall inception [26,15]. The duration of some peak events observed in the present work corresponds to 5 blades passing time or even more, therefore these could reduce the surge margin of the compressor according to Cousins [3]. The canonical configuration investigated is typical of the arrangements that have been previously studied to evaluate flow distortion in the absolute frame of reference. It is acknowledged that for a coupled intake and fan configurations the impact of the centrebody, and the presence of the fan, will affect the flow distortion. For example, it is expected that the level of unsteady distortion will reduce due to the axial flow acceleration over the centrebody. Nevertheless, the aim of this work is to propose and demonstrate a new way of evaluating unsteady flow distortion. Within that context, the identification and the assessment of these peak events in terms of magnitude, extent and likelihood is the fulcrum of the contribution to knowledge provided by this method.

\section{Effect of inlet total pressure profile on flow distortion}

The inlet boundary conditions were previously found to influence the unsteady flow distortion at the exit of an Sduct [26,32]. Within this context, the proposed analysis method was used to evaluate the impact of different inlet total pressure profiles on the flow characteristics. A gauze screen installed at section 3 (Fig. 1) was used to produce a nonuniform total pressure profile at the inlet of the intake whose non-dimensional thickness varied from nominal conditions (axi-symmetric boundary layer with a $\delta / \mathrm{D}_{\text {in }}=0.04$ ) to Profile A (thick boundary layer at the bottom $\delta / \mathrm{D}_{\text {in }}$ = 0.332). Profile A represents a typical inlet condition of a full scale BWB aircraft with the engine mounted near the wing-body trailing edge. The velocities shown in Fig. 11 are normalized by the time-averaged, area-averaged out-ofplane velocity at the AIP $\left(\overline{\left\langle v_{z}^{\prime}\right\rangle}\right)$ of the baseline inlet configuration $\left(\delta / \mathrm{D}_{\text {in }}=0.04\right.$, Fig. 8$)$. The flow non-uniformity caused by the increased profile thickness does not affect the general flow topology of the out-of-plane velocity and the tangential velocity (Fig. 11a-b). However, the thicker profile promotes the separation at the first bend of the Sduct, and there is a growth of the contra-rotating vortices at the AIP (Fig. 11b). The mass flow non-uniformity favors 
the downward pitching flow of the S-duct which strengthens the secondary flows (Fig. 11b) and accentuates the momentum non-uniformity at the AIP (Fig. 11a). A more in-depth analysis has been presented by McLelland et al. [24] in which unsteadiness and spectral analysis have been assessed for this thicker inlet profile configuration. The distribution of the relative velocity remains broadly unaltered (Fig. 11c), since it is mainly influenced by the rotational speed of the rotor, which is an order of magnitude greater than the in-plane velocity components. With the inlet Profile A $\left(\delta / D_{\text {in }}=0.332\right)$, the time-averaged incidence angle (Fig. 11d) shows more pronouncedly the areas of positive and negative incidence of the baseline case (Fig. 8d). The peak time-averaged value increases from the range $\left[-2^{\circ},+11^{\circ}\right]$ to $\left[-5^{\circ},+13^{\circ}\right]$. The distribution and the range of the incidence angle compares well with previous investigation with similar inlet conditions [32] even though in the current work there is no interaction between the outlet flow and the compressor geometry. A similar range of incidence angles is also reported by Giuliani and Chen [26] with the same inlet total pressure profile. All of these works broadly agree on the distribution of positive and negative incidence angles in the same characteristic locations, especially near the hub, at the bottom dead center and to opposite lateral sides of the spinner.

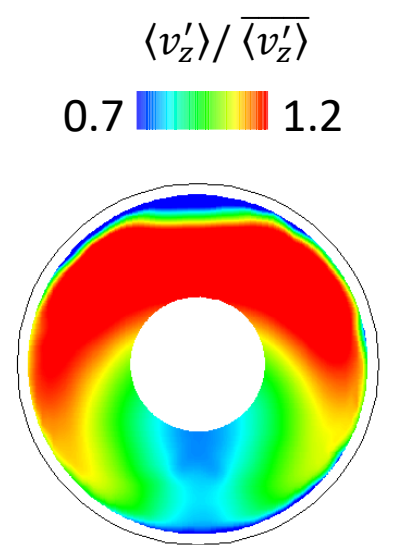

a)

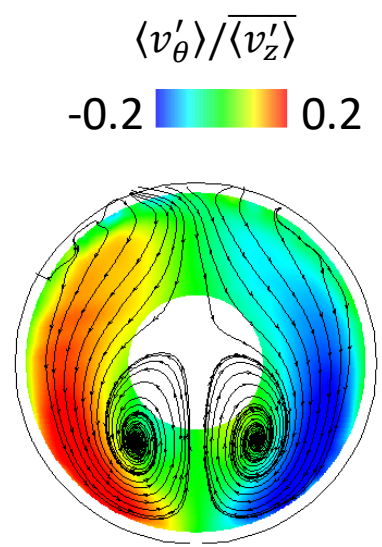

b)
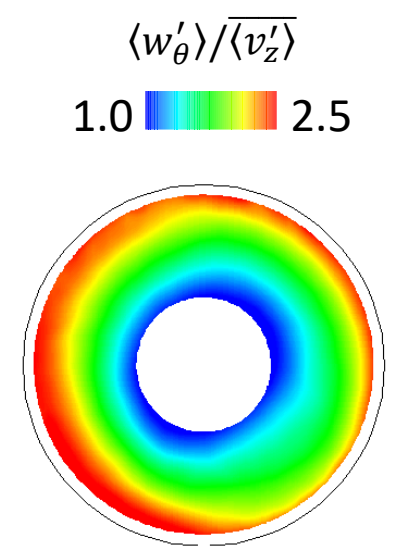

c) $\left\langle i^{\prime}\right\rangle\left(^{\circ}\right)$

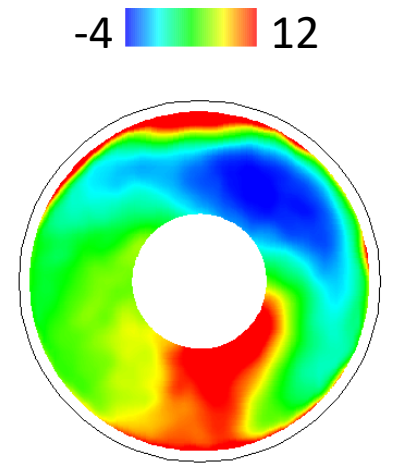

d)

Fig. 11: Time-averaged flow field with inlet Profile A $\left(\delta / \mathrm{D}_{\text {in }}=0.332\right)$.

The relative probability of the events has been computed with the normalization against the number of the events detected at the tip with baseline inlet conditions (Fig. 10a and b, $\delta / \mathrm{Din}=0.04)$. At the tip region $(\mathrm{r} / \mathrm{R}=0.85$ ), the nonuniform inlet total pressure profile (Profile $A, \delta / D_{\text {in }}=0.332$ ) causes an increase to the magnitude of the distortion events which are characterized by a longer exposure sector (Fig. 12a). For example, events with $120^{\circ}$ exposure sector have a mean incidence perturbation value $\left(\langle\Delta i\rangle_{e}\right)$ between $+14^{\circ}$ and $-15^{\circ}$, while the mean values were between $+9^{\circ}$ and $-13^{\circ}$ for the baseline inlet condition (Fig. 10a). In general, the increase of the mean incidence angle perturbations 
$\left(\langle\Delta i\rangle_{e}\right)$ of approximately $\pm 5^{\circ}$ with reference to the baseline case applies to all the events with exposure sector in the range between $60^{\circ}$ to $540^{\circ}$ (Fig. 12a). This increase in the magnitude of the incidence angle perturbation is also visible in the probability distribution of peak events for large exposure sector. For example, for exposure sector of about $5 t_{b p}$ the peak incidence perturbations increased from about $+15^{\circ}$ (Fig. 10b) to about $+20^{\circ}$ (Fig. 12b) with reference to the baseline case. However, in general, the thicker inlet profile (Profile $A, \delta / D_{\text {in }}=0.332$ ) seems to have a weak effect on the maximum exposure sector of the disturbances. Both the distribution of mean and peak incidence perturbations show a maximum exposure sector of about $540^{\circ}$ and about $150^{\circ}$ respectively (Fig. 12a, b), which is consistent with the maximum values for the baseline inlet conditions (Fig. 10a, b).

The increase of the boundary values of the mean incidence perturbations $\left(\langle\Delta i\rangle_{e}\right)$ is also visible in a position closer to the hub $(r / R=0.51$, Fig. 12c). For both maximum positive and negative incidence angle events, the increase of the absolute value is about $5^{\circ}$ (Fig. 12c) across the range of exposure sectors, in agreement to the observations at $\mathrm{r} / \mathrm{R}=0.85$. At the hub, some events with a long exposure sector of about $180^{\circ}$ and an incidence perturbation between $-5^{\circ}$ and $10^{\circ}$ showed a very high relative probability of about $1 \%$. The peak events distribution shows an increased probability of positive change of incidence events $\left(\Delta i_{\text {peak }}\right.$ ) (Fig. 12d), which can range from $12^{\circ}$ to over $35^{\circ}$ and a maximum exposure up to $15 t_{b p}$. In comparison, the range for baseline conditions was limited to $8^{\circ}$ to $34^{\circ}$ with exposure around $13 t_{b p}$ (Fig. 10d).

Summarizing, relative to the nominal inlet profile, the thicker inlet total pressure profile (Profile $A, \delta / \mathrm{D}_{\text {in }}=0.332$ ) caused an increase of positive incidence perturbations with long exposure sectors and an overall increased probability and magnitude of positive incidence perturbations. On the other hand, the likelihood and magnitude of the negative incidence perturbations are unaltered by the thicker inlet profile. This new analysis captures the variations in the distribution of mean and peak incidence perturbations for different inlet conditions. It can identify changes in exposure time and changes relative to the nominal incidence which are not possible to assess with conventional methods, which instead would detect only changes in the time-averaged quantities. 


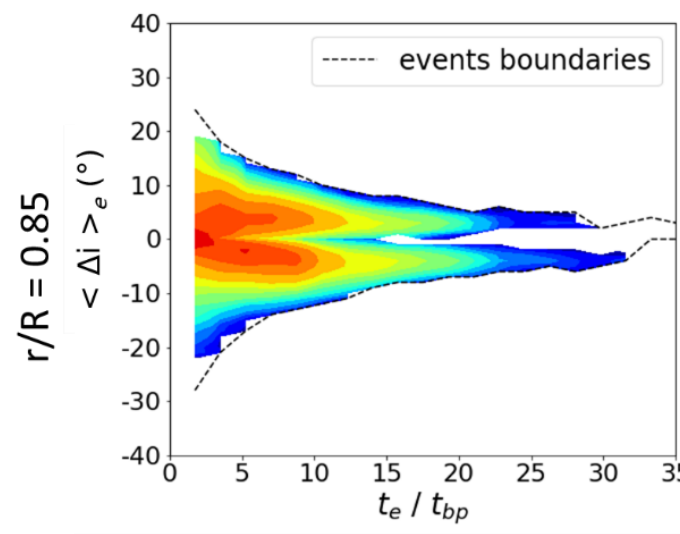

a)

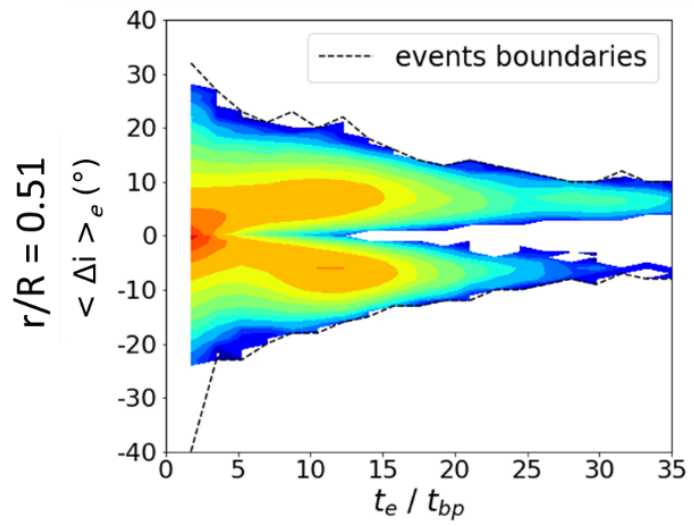

$0 \quad 60120180240300360420480540$ exposure sector $\left({ }^{\circ}\right)$

c)

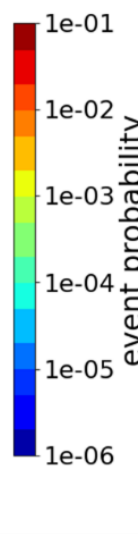

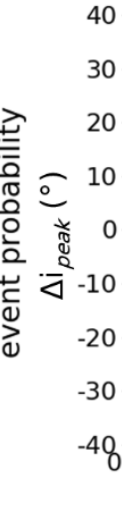

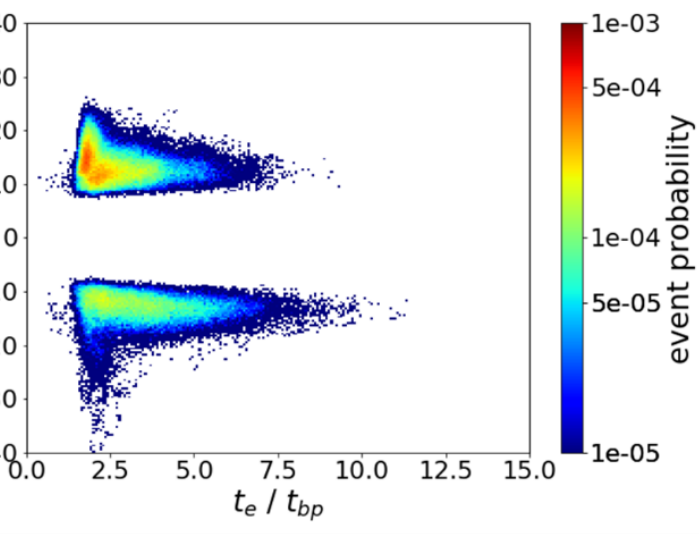

b)
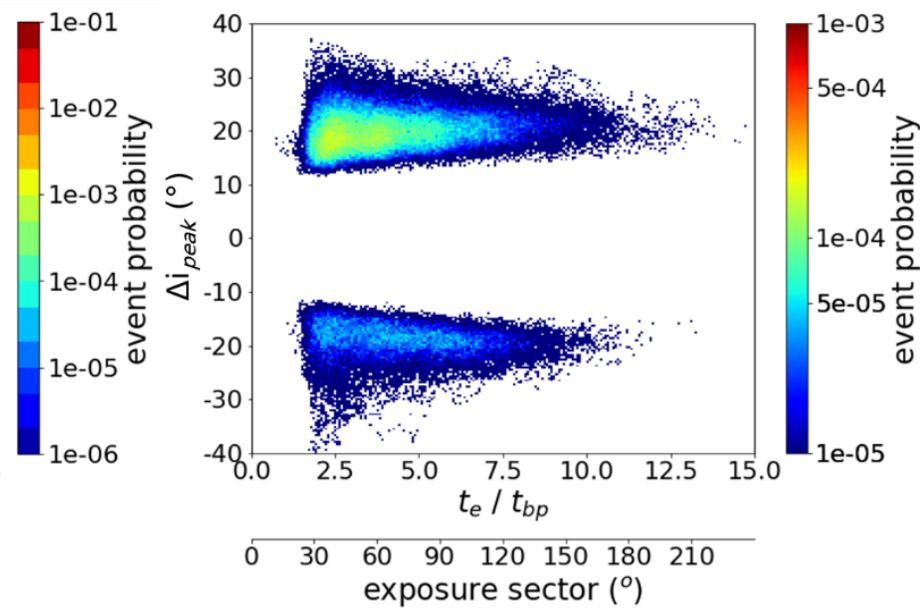
$030 \quad 60 \quad 90 \quad 120 \quad 150 \quad 180 \quad 210$
exposure sector $\left({ }^{\circ}\right)$

d)

Fig. 12: Probability maps for distortion events with inlet Profile $A\left(\delta / D_{i n}=0.332\right)$ for hub $(r / R=0.51)$ and tip $(\mathbf{r} / \mathbf{R}=\mathbf{0 . 8 5})$ positions. 


\section{Effect of inlet total pressure profile azimuthal orientation on distortion events}

To represent inlet conditions of an intake under angles of pitch and yaw the azimuthal orientation of the inlet flow Profile $\mathrm{A}\left(\delta / \mathrm{D}_{\text {in }}=0.332\right)$ was varied starting from the high pressure loss region at bottom dead center $\left(\psi=0^{\circ}\right)$ to angles of $\psi=45^{\circ}, 90^{\circ}$ (Fig. 1) [33]. These inlet conditions are expected when the duct axis is not aligned with the main fuselage body. One example is when the intake is located in a region of upper surface and spanwise flow causes skew in the airframe boundary layer or when the intake is operating at notable yaw angles for a top-mounted S-duct, or at notable pitch angles for a side-mounted S-duct [24,41].

Conventional studies would assess the impact of the inlet boundary layer orientation with the variations of the time-averaged incidence distributions $\left(\left\langle i^{\prime}\right\rangle\right)$ at the AIP (Fig. 13). For the baseline inlet conditions $\left(\delta / \mathrm{D}_{\text {in }}=0.04\right)$ and for Profile $\mathrm{A}\left(\delta / \mathrm{D}_{\text {in }}=0.332\right)$ at $\psi=0^{\circ}$, the area in the lower part of the domain shows peak positive incidence angles, with a quite symmetrical distribution with reference to the vertical axis (Fig. 13a, b). For a rotation of the Profile A to $\psi=45^{\circ}$ and $90^{\circ}$, this area of peak positive incidence appears closer to the hub and moves to the left of the domain (Fig. 13c, d). However, it is not clear from this distribution if the orientation of the thicker inlet profile has an impact on the operating conditions of the rotor. From the time-averaged distribution, it can be stated that, across the different configurations, Profile A $\left(\delta / \mathrm{D}_{\text {in }}=0.332\right)$ at $\psi=0^{\circ}$ is the inlet condition which generates the wider range of incidence angle $\left\langle i^{\prime}\right\rangle$. The range goes from $-5^{\circ}$ up to $+13^{\circ}$ and therefore this is the worst operating condition of the rotor. The application of the distortion events identification method aims to demonstrate the validity of this assumption.

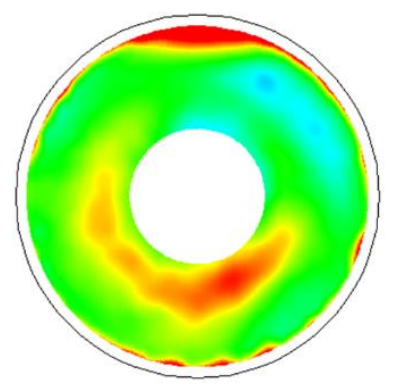

$$
\begin{gathered}
\text { Baseline } \\
\delta / D_{\text {in }}=0.04
\end{gathered}
$$
a)

$$
\left\langle i^{\prime}\right\rangle\left({ }^{\circ}\right)-4 \|
$$
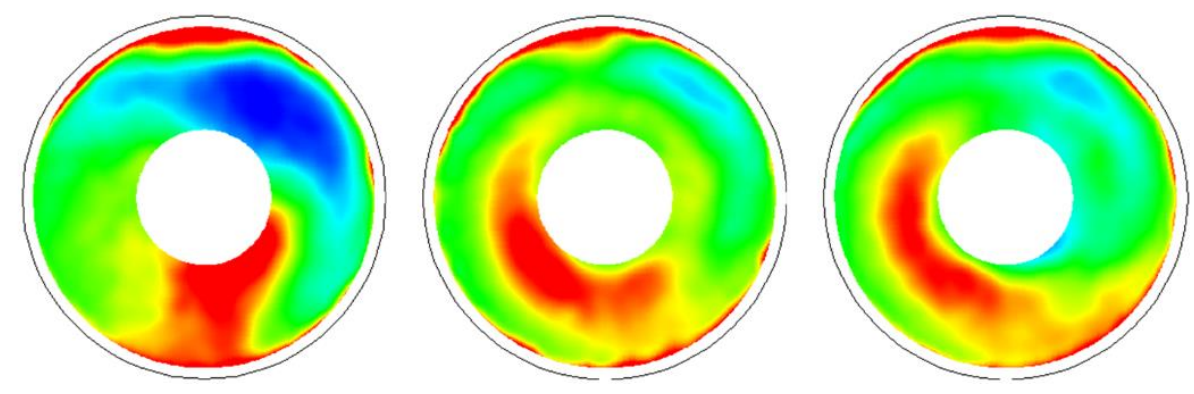

$$
\begin{gathered}
\delta / D_{\text {in }}=0.332 \\
\psi=0^{\circ}
\end{gathered}
$$

b)

$$
\begin{gathered}
\delta / D_{\text {in }}=0.332 \\
\psi=45^{\circ}
\end{gathered}
$$

c)

$$
\begin{gathered}
\delta / D_{\text {in }}=0.332 \\
\psi=90^{\circ}
\end{gathered}
$$

d)

Fig. 13: Time-averaged incidence angle distribution for baseline inlet condition $\left(\delta / D_{\text {in }}=0.04\right)$ and of inlet Profile $A\left(\delta / D_{\text {in }}=0.332\right)$ at different orientations. 
The rotation of the inlet Profile A by $\psi=45^{\circ}$ produced limited effects on the mean and peak values probability distributions if compared to the $\psi=0^{\circ}$ case (Fig. 14). As for Fig. 12, the probability of the events in Fig. 14 has been computed with the normalization against the number of the events detected at the tip with baseline inlet conditions (Fig. 10a and $\mathrm{b}, \delta / \operatorname{Din}=0.04)$. The maximum values of the mean incidence perturbations $\left(\left\langle\Delta i^{\prime}\right\rangle_{e}\right)$ are reduced by about $5^{\circ}$ in comparison with the $\psi=0^{\circ}$ orientation. For $\psi=45^{\circ}$, there is evidence of slightly higher peak events exposure sectors which maximum increases from $150^{\circ}\left(\psi=0^{\circ}\right.$, Fig. 14b) to about $180^{\circ}\left(\psi=45^{\circ}\right.$, Fig. $\left.14 \mathrm{c}\right)$, but the maximum value of incidence angle perturbations remains unchanged.

When the inlet Profile A was positioned at $\psi=90^{\circ}$, there is a more pronounced effect on the distribution of the peak events, which for the $\psi=90^{\circ}$ configuration are characterized by a $5^{\circ}$ reduction on average in maximum magnitude with reference to the $\psi=0^{\circ}$ inlet profile orientation (Fig. 14b, d). For the $\psi=90^{\circ}$ configuration, the event with the highest mean incidence reduced from $+29^{\circ}$ to $+23^{\circ}$ if compared with the inlet profile oriented at $\psi=45^{\circ}$. Beside the reduction of peak incidence perturbation magnitude, the probability distribution of the peak events reveals that the probability of positive extreme events with relatively large exposure sector increases when the inlet profile is at $\psi=90^{\circ}$ (Fig. 14d). For example, the relative probability for positive incidence perturbations of about $12^{\circ}$ and exposure sector of $60^{\circ}$ increases from 1/20,000 (Fig. 14b) to about 1/5,000 (Fig. 14d) when moving from Profile A at $\psi=0^{\circ}$ to $\psi=90^{\circ}$. Therefore, the inlet flow profile orientation has an impact on the most probable events, which are contained in a region close-to-zero incidence and an exposure sector up to $60^{\circ}$. Although the magnitude of these events is low compared to the maximum mean and peak values of the events at the boundaries, the high probability and significant exposure sector could severely affect the performance and the stability of the propulsion system.

In conclusion, this analysis revealed that the configuration with Profile $\mathrm{A}$ at $\psi=0^{\circ}$ does not necessarily generate the most challenging operating conditions for the compressor rotor, as previously inferred from the time-averaged incidence angle distributions (Fig. 13). Instead, the orientation of the inlet profile at $\psi=90^{\circ}$ could notably increase the probability of peak positive incidence perturbations at which the rotor blades are exposed for a longer time.

This new analysis also reveals further insights on the effects of boundary layer orientation on the S-duct flow distortion. For example, previous research [33] showed that the orientation of the inlet total pressure profile had a notable impact on the swirl patterns that develop at the AIP. However, it was not clear if this had also an impact on the duration of the distortion events on the rotor blades. With this new analysis it is possible to outline a better understanding of the complex unsteady flow distortion and the impact of the inlet boundary layer orientation. In 
addition, although previous analysis showed that peak swirl events were almost insensitive to a boundary layer orientation [33], this work revealed a reduction on the magnitude of peak and mean incidence perturbations for $\psi=$ $90^{\circ}$.

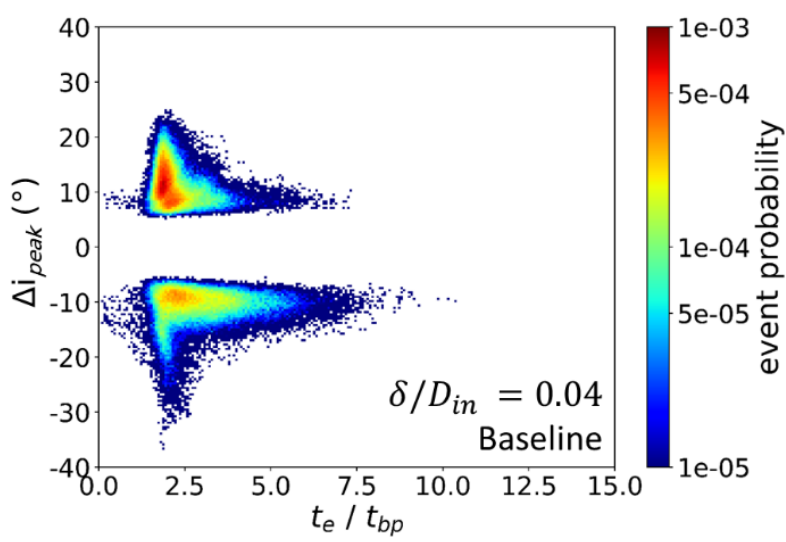

a)

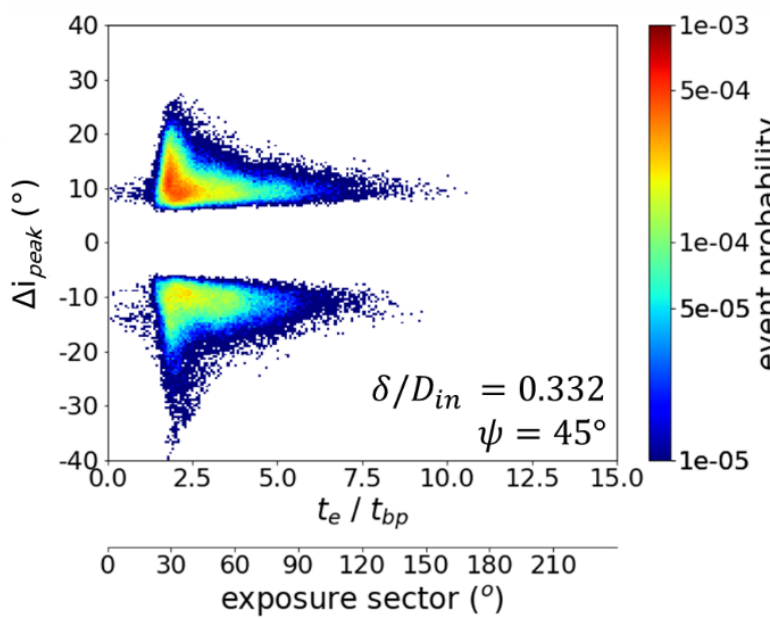

c)

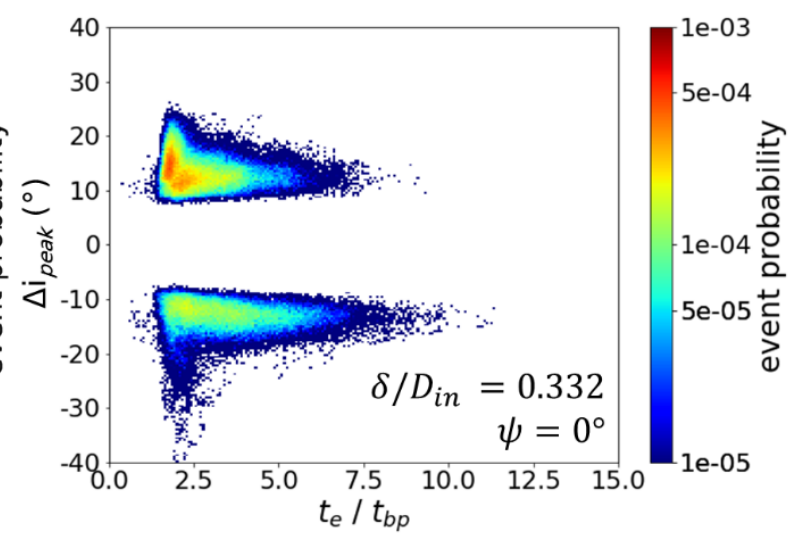

b)

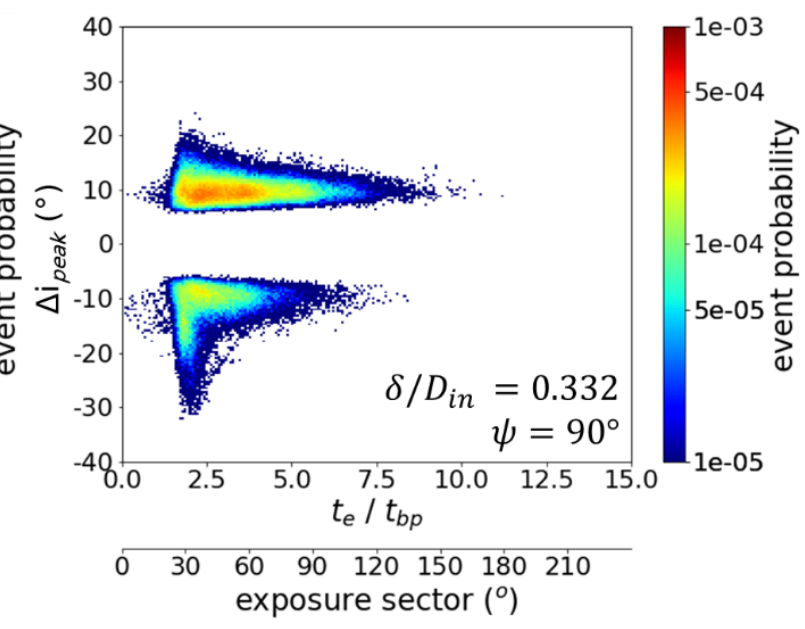

d)

Fig. 14: Probability maps for peak distortion events with baseline inlet conditions $\left(\delta / \mathrm{D}_{\text {in }}=0.04\right)$ and inlet Profile $A\left(\delta / D_{\text {in }}=0.332\right)$ with different orientations at $r / R=0.85$. 


\section{Conclusions}

A new approach for the evaluation of the unsteady intake flow distortion for aero-engine applications has been proposed. This novel approach takes into account the magnitude, the extent and the likelihood of the unsteady distortion events as would be presented to a rotating compression system. The analysis assessed the flow distortion at the outlet of an S-duct intake considering both the unsteady nature of the flow and also the variation produced by the change in position of the nominal rotor in the time-varying distorted flow field. Distortion events were identified based on the fluctuations of the incidence angle perturbations in the envisaged relative frame of reference and were classified by the time the blade was exposed to the distortion event, the change in incidence and the probability of the distortion events.

For the baseline inlet total pressure profile, the analysis revealed large fluctuations of incidence in the approaching conditions for a notional compressor. Some of the detected distortion events were found to be within the duration range of interest for stability assessment of an aero-engine subject to swirl distortion in terms of blade passing time.

The analysis also revealed that the probability distribution and the magnitude of the distortion events depend on the radial position at which the change in incidence is evaluated. A noticeable tendency towards positive incidence perturbations was observed at the tip while at the hub the events were distributed more equally between positive and negative incidence. On average across the range of exposure sectors, the magnitude of the mean incidence perturbations was $5^{\circ}$ greater at the hub.

Thicker inlet total pressure profiles were found to have a strong influence on the likelihood and the magnitude of distortion events with long exposure sector. The magnitude of peak and mean incidence perturbations of these events increased by approximately $5^{\circ}$ in comparison with the baseline case, independently of the events' duration. At the hub, the probability of positive incidence perturbations increased in comparison to the baseline inlet conditions. Generally, thicker inlet boundary layers had only a limited impact on the maximum exposure time of the distortion events. Different operating conditions of the S-duct intake were re-created by changing the azimuthal position of the inlet boundary layer. The change in position from $\psi=0^{\circ}$ to $45^{\circ}$ and $90^{\circ}$ reduced the magnitude of the peak and mean incidence perturbations. However, the peak events identification revealed that for $\psi=90^{\circ}$ there was a substantial increase of positive incidence angle perturbation with large exposure sectors.

The novel method proposed in this work was able to assess for the first time how nominal rotor blades were exposed to incidence angle perturbations and quantified which is the likelihood of extreme events which could cause 
stability issues in the propulsion system. The focus of the investigation moved away from the time-averaged incidence angle distribution typical of conventional methods in favor of the identification of unsteady distortion events. This method demonstrated that the time-averaged incidence angle distribution obscured the large fluctuation and the peak values of the unsteady incidence perturbation on the blades. It also quantified the increase in exposure time for the distortion events and estimated the peak values of incidence perturbations for a range of different inlet conditions. Thus, it also proved to be successful for the application to different test cases such as non-uniform inlet conditions.

\section{Funding Sources}

Mr. Migliorini is partially funded by UK Engineering and Physical Sciences Research Council (UKRI), award reference no. 2202953. Due to commercial confidentiality agreements the supporting data is not available.

\section{References}

[1] Kurzke, J., and Halliwell, I., "Propulsion and Power,” 1st ed., Springer International Publishing, Cham, 2018. doi: 10.1007/978-3-319-75979-1.

[2] “A Methodology for Assessing Inlet Swirl Distortion,” Automotive Engineers Aerospace Information Rept. AIR 5686, Warrendale, PA, 2007.

[3] Cousins, W. T., "History, Philosophy, Physics, and Future Directions of Aircraft Propulsion System / Inlet Integration," Proceedings of the ASME Turbo Expo, GT2004-54210, Vol. 2, ASME, Vienna, 2004, pp. 305-320. doi: $10.1115 / G T 2004-54210$

[4] Oates, G. C., “Axial Flow Compressor Aerodynamics," Aerothermodynamics of Aircraft Engine Components, AIAA Education Series, AIAA, New York, 1985, pp. 145-218.

[5] Provenza, A. J., Duffy, K. P., and Bakhle, M. A., “Aeromechanical Response of a Distortion-Tolerant Boundary Layer Ingesting Fan,” Journal of Engineering for Gas Turbines and Power, Vol. 141, No. 1, Jan. 2019.

doi: $10.1115 / 1.4040739$

[6] Hodder, B. K., “An investigation of engine influence on inlet performance,” NASA CR-166136, Seattle, WA, United States, 1981.

[7] Larkin, M. J., and Schweiger, P. S., "Ultra high bypass Nacelle aerodynamics inlet flow-through high angle of attack distortion test," NASA CR-189149, United States, 1992.

[8] Carnevale, M., Wang, F., and di Mare, L., "Low Frequency Distortion in Civil Aero-engine Intake," Journal of Engineering for Gas Turbines and Power, Vol. 139, No. 4, Apr. 2017, doi: 10.1115/1.4034600. 
[9] Shaw, M. J., “An Assessment of CFD for Transonic Fan Stabilities Studies,” Ph. D. dissertation, Queens' College, University of Cambridge, 2015.

[10] Zhang, W., and Vahdati, M., “A Parametric Study of the Effects of Inlet Distortion on Fan Aerodynamic Stability,” Journal of Turbomachinery, Vol. 141, No. 1, Jan. 2019, p. 011011. doi: $10.1115 / 1.4041376$.

[11] Mailach, R., Lehmann, I., and Vogeler, K., "Periodical Unsteady Flow Within a Rotor Blade Row of an Axial CompressorPart I: Flow Field at Midspan,” Journal of Turbomachinery, Vol. 130, No.4, 2008, p. 041004. doi: $10.1115 / 1.2812329$.

[12] Freeman, C., and Rowe, A. L., "Intake Engine Interactions of a Modern Large Turbofan Engine," Proceedings of the ASME 1999 International Gas Turbine and Aeroengine Congress and Exhibition, Volume 1: Aircraft Engine; Marine; Turbomachinery; Microturbines and Small Turbomachinery, 99-GT-344, ASME, Indianapolis, US, 1999. https://doi.org/10.1115/99-GT-344

[13] Vadlamani, N. R., Cao, T., Watson, R., and Tucker, P. G., "Toward future installations: Mutual interactions of short intakes with modern high bypass fans," Journal of Turbomachinery, Vol. 141, No. 8, July 2019, p. 081013. doi: $10.1115 / 1.4044080$.

[14] Longley, J. P., Shin, H.-W., Plumley, R. E., Silkowski, P. D., Day, I. J., Greitzer, E. M., Tan, C. S., and Wisler, D. C., “Effects of Rotating Inlet Distortion on Multistage Compressor Stability,” Journal of Turbomachinery, Vol. 118, No. 2, Apr. 1996, pp. $181-188$.

doi: $10.1115 / 1.2836624$

[15] Tan, C. S., Day, I., Morris, S., and Wadia, A., "Spike-Type Compressor Stall Inception, Detection, and Control,” Annual Review of Fluid Mechanics, Vol. 42, Jan. 2010, pp. 275-300, doi: 10.1146/annurev-fluid-121108-145603

[16] Wenzel, L. M.; Blaha, R. J., “Analysis of Dynamic Inlet Distortion Applied to a Parallel Compressor Model,” NASA-TMX-3522, Washington D.C., US, 1977.

[17] Silkowski, P. D., "Measurements of Rotor Stalling in a Matched and a Mismatched Multistage Compressor," GTL-221GTL, Gas Turbine Laboratory, Massachusetts Institute of Technology, Cambridge, MA, 1995.

[18] Zachos, P. K., MacManus, D. G., Prieto, D. G., and Chiereghin, N., "Flow Distortion Measurements in Convoluted Aeroengine Intakes," AIAA Journal, Vol. 54, No. 9, Sep. 2016, pp. 2819-2832.

doi: 10.2514/1.J054904 
[19] Tanguy, G., MacManus, D. G., Garnier, E., and Martin, P. G., "Characteristics of Unsteady Total Pressure Distortion for a Complex Aero-engine Intake Duct,” Aerospace Science and Technology, Vol. 78, Jul. 2018, pp. 297-311.

doi: 10.1016/j.ast.2018.04.031

[20] Gil-Prieto, D., MacManus, D. G., Zachos, P. K., Tanguy, G., and Menzies, K. R., “Convoluted Intake Distortion Measurements Using Stereo Particle Image Velocimetry,” AIAA Journal, Vol. 55, No. 6, Jun. 2017, pp. 1878-1892. doi: $10.2514 / 1 . J 055467$

[21] Holmes, P., Lumley, J. L., and Berkooz, G., "Turbulence, Coherent Structures, Dynamical Systems and Symmetry," Cambridge Monographs on Mechanics, Cambridge University Press, Cambridge, 1996.

[22] Tanguy, G., MacManus, D. G., Zachos, P., Gil-Prieto, D., and Garnier, E., "Passive Flow Control Study in an S-Duct Using Stereo Particle Image Velocimetry,” AIAA Journal, Vol. 55, No. 6, Jun. 2017, pp. 1862-1877. doi: 10.2514/1.J055354

[23] Gil-Prieto, D., Zachos, P. K., MacManus, D. G., and McLelland, G., "Unsteady Characteristics of S-duct Intake Flow Distortion,” Aerospace Science and Technology, Vol. 84, Jan. 2019, pp. 938-952. doi: 10.1016/j.ast.2018.10.020

[24] McLelland, G., MacManus, D. G., Zachos, P. K., Gil-Prieto, D., and Migliorini, M., "Influence of Upstream Total Pressure Profiles on S-Duct Intake Flow Distortion,” Journal of Propulsion and Power, Vol. 36, No. 3, May 2020, pp. 346-356. doi: 10.2514/1.B37554.

[25] Jirásek, A., "Example of Integrated Computational Fluid Dynamics and Experimental Studies: Design of Flow Control in the FOI-EIC-01 Inlet," Proceedings of the Institution of Mechanical Engineers, Part G: Journal of Aerospace Engineering, Vol. 223, No. 4, Apr. 2009, pp. 369-377. doi: 10.1243/09544100JAERO390.

[26] Giuliani, J. E., and Chen, J.-P., "Fan Response to Boundary-Layer Ingesting Inlet Distortions," AIAA Journal, Vol. 54, No. 10, Oct. 2016, pp. 3232-3243 doi: $10.2514 / 1 . J 054762$

[27] Defoe, J. J., and Spakovszky, Z. S., "Effects of Boundary-Layer Ingestion on the Aero-Acoustics of Transonic Fan Rotors," Journal of Turbomachinery, Vol. 135, No. 5, Sep. 2013, pp. 51013-51018. doi: $10.1115 / 1.4023461$

[28] “Inlet Total-Pressure-Distortion Cosiderations for Gas-Turbine Engines," Aerospace Information Rept. AIR 1419A, Society of Automotive Engineers, 1999.

[29] MacManus, D. G., Chiereghin, N., Prieto, D. G., and Zachos, P., "Complex Aeroengine Intake Ducts and Dynamic Distortion," AIAA Journal, Vol. 55, No. 7, Jul. 2017, pp. 2395-2409. 
doi: $10.2514 / 1 . J 054905$

[30] Bravo-Mosquera, P. D., Abdalla, A. M., Cerón-Muñoz, H. D., and Martini Catalano, F., "Integration assessment of conceptual design and intake aerodynamics of a non-conventional air-to-ground fighter aircraft," Aerospace Science and Technology, Vol. 86, Mar. 2019, pp. 497-519, doi: 10.1016/j.ast.2019.01.059.

[31] Gil-Prieto, D., MacManus, D. G., Zachos, P. K., Tanguy, G., Wilson, F., and Chiereghin, N., "Delayed Detached-Eddy Simulation and Particle Image Velocimetry Investigation of S-Duct Flow Distortion,” AIAA Journal, Vol. 55, No. 6, June 2017, pp. 1893-1908. doi: $10.2514 / 1 . J 055468$.

[32] Gunn, E. J., and Hall, C. A., “Aerodynamics of Boundary Layer Ingesting Fans," Proceedings of the ASME Turbo Expo 2014: Turbine Technical Conference and Exposition. Volume 1A: Aircraft Engine; Fans and Blowers, GT2014-26142, ASME, Düsseldorf, Germany, 2014.

doi: $10.1115 / G T 2014-26142$

[33] Migliorini, M., Zachos, P., and MacManus, D., “The Impact of Inlet Boundary Layer Thickness on the Unsteady Aerodynamics of S-duct Intakes," AIAA Propulsion and Energy 2019 Forum, AIAA Paper 2019-4202, AIAA, Indianapolis, IN, 2019.

doi: 10.2514/6.2019-4202

[34] Rein, M., and Koch, S., "Experimental Study of Boundary-Layer Ingestion into a Diverterless S-Duct Intake," AIAA Journal, Vol. 53, No. 11, Nov. 2015, pp. 3487-3492.

doi: 10.2514/1.J053902

[35] Garnier, E., "Flow Control by Pulsed Jet in a Curved S-Duct: A Spectral Analysis," AIAA Journal, Vol. 53, No. 10, October 2015, pp. 2813-2827.

doi: 10.2514/1.J053422

[36] Raffel, M., Willert, C. E., Wereley, S. T., Kompenhans, J., Willert, S., Wereley, S. T., and Kompenhans, J., "Particle Image Velocimetry: A Practical Guide,” Particle Image Velocimetry, Springer-Verlag, ed., Springer, Berlin, 2007, pp. $203-241$.

[37] Owens, L. R., Allan, B. G., and Gorton, S. A., "Boundary-Layer-Ingesting Inlet Flow Control,” Journal of Aircraft, Vol. 45, No. 4, Jul. 2008, pp. 1431-1440. doi: $10.2514 / 1.36989$

[38] Strazisar, A. J., and Powell, J. A., "Laser Anemometer Measurements in a Transonic Axial Flow Compressor Rotor,” NASA TM-79323, Cleveland, OH, 1979. 
[39] Dean, W. R., "XVI. Note on the Motion of Fluid in a Curved Pipe," The London, Edinburgh, and Dublin Philosophical Magazine and Journal of Science, Vol. 4, No. 20, Jul. 1927, pp. 208-223. doi: $10.1080 / 14786440708564324$

[40] Medhi, A., "Effect of Swirl Distortion on Gas Turbine Operability," Ph. D. dissertation, Propulsion Engineering Centre, School of Aerospace, Transport and Manufacturing, Cranfield University, 2014.

[41] Hall, D. K., Huang, A. C., Uranga, A., Greitzer, E. M., Drela, M., and Sato, S., "Boundary Layer Ingestion Propulsion Benefit for Transport Aircraft," Journal of Propulsion and Power, Vol. 33, No. 5, Mar. 2017, pp. 1118-1129. doi: 10.2514/1.B36321. 
2021-09-13

Novel method for evaluating intake unsteady flow distortion

\author{
Migliorini, Matteo
}

AIAA

Migliorini M, Zachos PK, MacManus DG. (2022) Novel method for evaluating intake unsteady pÿflow distortion. Journal of Propulsion and Power, Volume 38, Number 1, January February 2022, pp. 135-147

https://doi.org/10.2514/1.B38127

Downloaded from Cranfield Library Services E-Repository 